\title{
Application of Data Clustering to Railway Delay Pattern Recognition
}

\author{
Fabrizio Cerreto $\left(\mathbb{D},{ }^{1}\right.$ Bo Friis Nielsen, ${ }^{2}$ Otto Anker Nielsen, ${ }^{1}$ and Steven S. Harrod ${ }^{1}$ \\ ${ }^{1}$ Department of Management Engineering, Technical University of Denmark, 2800 Kongens Lyngby, Denmark \\ ${ }^{2}$ Department of Applied Mathematics and Computer Science, Technical University of Denmark, 2800 Kongens Lyngby, Denmark \\ Correspondence should be addressed to Fabrizio Cerreto; facer@dtu.dk
}

Received 21 November 2017; Revised 20 February 2018; Accepted 11 March 2018; Published 29 April 2018

Academic Editor: Andrea D’Ariano

Copyright (C) 2018 Fabrizio Cerreto et al. This is an open access article distributed under the Creative Commons Attribution License, which permits unrestricted use, distribution, and reproduction in any medium, provided the original work is properly cited.

\begin{abstract}
$K$-means clustering is employed to identify recurrent delay patterns on a high traffic railway line north of Copenhagen, Denmark. The clusters identify behavioral patterns in the very large ("big data") datasets generated automatically and continuously by the railway signal system. The results reveal the conditions where corrective actions are necessary, showing the cases where recurrent delay patterns take place. Delay profiles and delay change profiles are generated from timestamps to compare different train runs and to partition the set of observations into groups of similar elements. $K$-means clustering can identify and discriminate different patterns affecting the same stations, which is otherwise difficult in previous approaches based on visual inspection. Classical methods of univariate analysis do not reveal these patterns. The demonstrated methodology is scalable and can be applied to any system of transport.
\end{abstract}

\section{Introduction}

Operations analysis is the collection and review of performance data, such as punctuality and process cycle time. It is a key step in the continuous improvement of transport services, and several methods exist to collect and analyze data from operations. The increasing availability of automated data sources is offering new ways to analyze operations, providing deeper insight and more reliable information. Railway management is very accepting of these new possibilities, and considerable effort is made by operators and institutions to use operations analysis in feedback loops for improving the timetabling process [1-4]. A better understanding of the development of delays in railways, and in transportation in general, provides the opportunity to improve the processes and identify the factors affecting reliability. For example, causes of delays might be identified in misallocation of supplements and buffers in timetables, structural conflicts that require mitigation actions, suboptimal design of station processes, and inefficient procedures for preparing a train for departure. This paper demonstrates a data mining technique based on $k$-means clustering to identify recurrent delay patterns in transportation, identify the main reason for cluster membership, and provide managerial insight to improve timetables and processes.

Prior studies propose several methods that are currently in use for operation analysis, deploying sources of automatic data collection. These approaches can be divided into traditional statistical methods and big data techniques, which differ in both the use of data and in the output provided. Traditional methods tend to aggregate and summarize information, so these can provide a general picture or detailed information on specific stations or trains. These are typically proposed in the form of multiple univariate distribution analysis, where the occurrence of different delay patterns at the same station is not visible. Big data techniques can be used to investigate recurring patterns or internal structures in operations. These approaches are expanding, thanks to the growing availability of large amounts of data, and several techniques have been deployed to identify recurrences of delays and describe or predict delays. Advanced techniques such as neural networks, succession rules, Bayesian networks, and various methods of regression have been developed mainly to predict real time delays in railways, as described 
in Section 2. However, train delays are necessarily correlated over the progression of a complete journey, and these data relations both along the journey of a train and among adjacent train paths have not received as much attention in the literature.

This paper presents a big data technique to identify recurring delay patterns in railway operations. Big data refer to information assets characterized by high volume, velocity, and variety, whose value is extrapolated by analytical methods [5]. In this application, the absolute delay and delay change are tracked for individual train paths along a railway line, resulting in absolute delay and delay change profiles. In the papers based on univariate statistics, systematic delays in these profiles are identified through visual inspection. The manual search for similarities suffers from subjective interpretation from the operator and is easily biased by common artefacts of the representation. The technique presented in this paper applies $k$-means clustering to find recurrent patterns in train delay progression, so that management may identify processes for improvement or correction. In this way it is possible to support continuous quality improvement.

In Section 2, a literature survey of contemporary data analysis methods is offered. Section 3 presents the $k$-means cluster method and the structure of the data to be studied. Section 4 presents results from the study of a high density Danish railway line. The effectiveness of $k$-means clustering for this application is discussed in Section 5, particularly with regard to its novelty compared to existing literature, while conclusions of this paper are presented in Section 6.

\section{Literature Survey}

Operations analysis is fundamental in the continuous improvement process to manage and modify railway operations. Data collected from real operations, or from simulation models, has been used in the feedback loop to design and improve railway timetables for decades. Typically, even if timetables may change over time, some of the fundamental infrastructure and service behaviors will not be modified. Timetables are often the result of only minor modifications to the previous editions and need to consider problems discovered in earlier timetables. For example, after a structural change in the Danish railway timetable in 1998, after the opening of the Great Belt fixed link, the service structure remained largely unchanged until 2016 [6].

Data collection systems have proliferated in railway networks since 2000, and very large amounts of data are available today. Widespread systems to collect data increased both volume and variety of data, which are often collected by different systems at the same time. The methods to elaborate and interpret information from past operations evolved together with the amount and quality of data, starting from descriptive and inferential statistic and moving towards big data techniques. For example, delay probability density functions can be extrapolated from historical data and integrated in analytical models to estimate service reliability before operation [7]. Goverde et al. [8] perform extensive statistical analysis and distribution fitting of data from the Dutch railway network. Goverde et al. fit different distributions for arrival and departure delays and find that no general distribution fits all groups of recorded arrival delays.

Primary delay distributions derived from operational data are also often employed as input in simulation models to evaluate the propagation of delays. Sipilä [9] explores the effect of modified running time supplements in railway schedules through microsimulation of a Swedish railway line. The author identifies different strategies for running time supplement allocation by verifying the significance of the change in punctuality recorded in 1600 simulations of selected scenarios. Lindfeldt [10] describes a method to aggregate delay data from real records and isolate distributions of primary delays. These distributions are then used to formulate microsimulation models. The data consists of manual records from dispatchers that assign a delay cause code to every record greater than 4 minutes of delay on the Swedish railways. In absence of other sources of data, the reliability of manual record cannot be validated, although the whole simulation model and its results rely on the derived distributions. Studies from other countries show that manual input can be indeed unreliable [11, 12]. The same method to extract primary delay distributions is later used by Lindfeldt and Sipilä [13] in a simulation model to assess the effect of allowing freight trains to travel outside their assigned path. The authors demonstrate that the realized travel times of freight trains could be shortened considerably without affecting the performance of other trains. The reduction of unnecessary waits for traffic management and the permission to depart before schedule reduced the average travel time on one side but increased its variability on the other.

Historical data also provides insight into the factors that influence service reliability. Olsson and Haugland [14] apply regression analysis on the Norwegian railway network and identify the most relevant factors for punctuality, such as absolute passenger flow and passenger occupation ratio. Gorman [15] uses regression analysis on data from American single-tracked freight railways to identify the factors that contribute the most to prolongation of railway running times. Gorman predicts congestion delay based on meets and passes scheduled as a consequence of speed heterogeneity. Again, in simulation, Shih et al. [16] apply an approach similar to Gorman's to determine the best capacity expansion strategy in terms of reduction of average prolongation of running time for freight trains. Shih et al. identify functional relationships, through regression of simulation results, between average delay per train-mile and several factors, such as the relative length of the doubled-tracked section of a railway line. Lindfeldt [17] applies multilinear regression with special focus on $F$-statistics to investigate factors generating delays on the Swedish railway network. Lindfeldt measures delay changes over selected routes and analyzes their distributions. In particular, the response variables are the share of trains with a delay increase, the median change in delay, and its standard deviation on the route. Statistically significant explanatory variables are found in the traffic volume for both passenger and freight trains. Among passenger trains the most significant variables are average speed and traffic 
heterogeneity, and for freight trains it is the number of stations on the route with at least three tracks.

Timestamps and recorded deviations from schedule can be integrated with information from other sources. For example, incident reports may be compiled in case of larger disruptions. Such reports include information about the typology of the incident, the train affected by the primary delay, other trains involved, the secondary delays generated, and the recovery plans taken by the dispatchers. Schittenhelm and Richter [1] describe the reporting system in the Danish railways (the same system in service at the time of this study) and introduce a quantile-based approach to depict the development of train delays en route. The plots confirm the general understanding of delays from experienced operators and can be used to quantify the magnitude of expected disruption. The quantile-based approach, though, describes operations as a whole, and it is not able to distinguish systematic delays occurring at individual stations, but with different origins, so analysis of individual train services is necessary to identify peculiar delay patterns. Richter [18] introduces new metrics to identify improvement actions, based on data from automatic detection systems. Richter sorts the trains according to recorded delay and identifies the worst one in a percentile approach, associated with recorded delay causes. A similar approach is adopted with regard to change in deviation, or delay jump, recorded on line sections, so that most critical geographical areas are identified. Lastly, Richter proposes a tabular representation of the median delay of individual trains recorded at station, sorted by scheduled time and geographical location. In this way, the analyst can identify which specific trains typically suffer from primary delays, also characterized by geographical location, and which trains are typically affected.

Similarly, Peterson [4] studies the on-time performance along the path of specific train services, using the rolling average delay of the last three timing points. Such on-time performance is plotted for all the repetitions of a specific train service over a time period and compared to the average, standard deviation, and 75th percentile. Peterson identifies empty areas in the pool of plotted delay profiles and interprets these as recurrent delay patterns given by discrete dispatching choices along the train path. Peterson also interprets recurrent increases or decreases of vehicle delay as segments of insufficient or excess running time supplement, respectively. Reliability of service is described by the standard deviation of recorded delays. Peterson used the mentioned measures in a feedback loop to redistribute the running time supplement in train paths according to the recorded performance.

Andersson et al. [19] assess the effectiveness of running time supplement in railway schedules from empirical data collected on a Swedish railway line. The study plots the recorded delays over the train itinerary overlapped with scheduled running time supplement and compares pairwise stacked plots from different railway services, stopping patterns, or directions. The identification of misallocation of running time supplement is based on visual search for recurrent delay patterns, and a few different dispatching tactics are identified in clusters of similar delay profiles. Andersson et al. highlight the existence of a threshold value of delay that triggers prioritization of other trains that are traveling on schedule. The observations are clustered in groups and show recurrent delay patterns, and the analysis is supported by detailed analysis of possible conflicts among individual train itineraries. Noticeably, the authors demonstrate that the measures of punctuality currently in use on the Swedish network hide the effects of running time supplement misallocation and delays developed en route. Even though the punctuality at the final destination is a measure of railway performance very common among railway operators, it does not express how trains increase or recover from delays along their journey. Schittenhelm [20] provides a sample of similar measuring approaches in the European railway industry. In a later study, Andersson et al. [21] underline the relevance of critical points for network robustness by plotting delay profiles and showing that the profiles cluster around critical points according to different dispatching strategies. Advanced clustering techniques may support the identification of different strategies to compute the effects on robustness.

Lastly, van Oort et al. [22] evaluate data collected automatically on public transport services with a combination of statistical methods and visual representation. The study represents delay data similarly to Peterson [4], Andersson et al. [19, 21], and Schittenhelm and Richter [1], plotting the recorded delay over individual repetitions of the same service path, and adds the plot of relevant delay percentiles over the stations. The shape of the percentile-based delay profiles highlights recurrent patterns in the deviation from schedule. The representative delay profiles appear different depending on the percentile they represent. Patterns found included the presence of typical early arrival at stations in bus services, followed by waiting time until the scheduled departure time, or recurrent delay drops or increases at specific stations. The delay plots are combined with the measured headway from the previous vehicle. While the delay plots would suggest allocating more running time supplements at systematic increases of delay, structural delays that cannot be compensated by timetable slack are highlighted in the plots of headways, where service unreliability corresponds to scattered recorded headways. A percentile approach was also presented by van Oort et al. to characterize and sort the stations according to performance, similarly to previous literature.

The statistical analyses presented above are suitable for general description of the system performance but lack specific insight into recurrent delay patterns that occur in operation and into the relationships between delays at different locations. The literature presented in this survey focuses on the univariate analysis of selected measures, such as delays at single stations. Traditional metrics common in the railway industry, such as punctuality, have also been found unrepresentative of the actual service reliability. The methods that include the multidimensional aspect of the problem mostly deal with delay profiles, the sequences of delays recorded on individual train itineraries. The quality of these analyses often relies on visual inspection of plotted data and the observer-operated search for matching delay profiles. This search lacks a standardized methodology and is influenced by 
the plotting layout and the subjective interpretation, which is based on personal experience.

Big data techniques have arisen recently and seek to make use of the very large amount of information that is provided by automatic data collection systems, overcoming the mentioned issues of traditional methods. The term big data is rather broad and includes different techniques that serve specific purpose. The common characteristics of these techniques are volume, velocity, and variety, meaning large amounts of data, generated at high speed, possibly by different sources with different or no structure [5]. As opposed to standard statistical analyses, where hypotheses are formulated and tested, big data techniques search for internal structures directly in the data. Data generated by automatic sources typically fit in the big data criteria. In railways, several data mining techniques were developed in the last years, following different approaches and searching for different types of information. The interest in these techniques is rising, together with the increasing availability of structured data. Industrial applications of these techniques are spreading, and new approaches are being studied also among public institutions [2].

Event mining is a technique based on time sorted logs, where relations between different events are found based on their coincidences. Hansen et al. [23] combine an event mining tool and standard statistics to predict the actual running times of trains to the next station, given all the recorded current delays. Dependencies between pairs of events are found or "mined" in timed event graphs created from the timestamps of individual trains, which correspond to events of occupation and release of blocking sections. The process times between events are inspected by standard statistics, resulting in conditional probabilities of process times, given the recorded delays of all relevant trains in the system. Such a model, though, relies considerably on very detailed knowledge about the infrastructure and requires data which is not commonly available from railway infrastructure managers.

Goverde and Meng [11] use the same information source and similar technique to identify and analyze route conflicts and identify delay chains. Infrastructure data and operation data are integrated so that it is possible to identify a train that is occupying a blocking section linked to a signal at danger for another train. Delay trees are built and traced backwards to identify the primary causes, so individual delays can be classified automatically into primary and secondary, and the correct attribution of delay causes can be verified. Interestingly, the authors verify that more than half of the delay cause records were assigned wrongly by the dispatchers, stating that, in the Netherlands, this type of manual input is not reliable and objective enough to be deployed in data analysis.

Kecman and Goverde [24] extend the model to include nonlogged line sections, where it is not possible to distinguish delays due to signaling impositions and delays due to primary causes. Delay chains are also traced in less detailed data by Sørensen et al. [12]. Based on the time sequences at stations experiencing disturbed operations, the authors identify the trains generating the conflicts and the trains suffering from the conflicts. The analysis is used to identify primary delays, describe single days of operation, identify frequent trains originating, or subject to, delay chains, and identify point stations where most of the primary or secondary delays are generated. In a comparison with manually recorded delay causes, the study finds relevant inconsistencies with the primary delays traced in the delay chains, in accordance with Goverde and Meng [11]. The method described, though, is only valid for single track lines and does not identify multiple primary delays.

Cule et al. [25] introduce association rules to identify delays recurring often together and set up an episode mining framework to highlight frequent delay patterns from train timestamps at stations. However, association rules can highlight common recurrences but cannot explain relations of causality between two events, so primary and secondary delays cannot be distinguished. Similarly, Wallander and Mäkitalo [26] identify delay chains according to the manual delay cause records from the dispatchers and based on timestamps at stations with granularity of 1 minute. The succession rules used are very similar to association rules but consider the time dependencies, so that events taking place earlier can be assumed to be the cause of events happening later under the same circumstances. Trains are characterized by the number and magnitude of conflicts they generate so that improvement actions can be concentrated. Association rules have also been adopted to evaluate the effectiveness of delay prevention actions on Japanese suburban networks by Yabuki et al. [27]. Yabuki et al. compare the association among occurrence of delays of different trains, change in delays, extension of running and dwelling times, and realized headway in before/after scenario comparison. The downside of such models is that association rules can be set between binary variables, so the development of delays depicted does not include its magnitude. Further, the number of associations to be analyzed grows exponentially with the number of potential pairs of events, so the analyses must be limited to short time frames of operation.

Neural networks are a big data method that learns from historical records and uses the relations identified among variables to predict an output, given unseen values of the input variables. This technique is particularly suited to delay prediction and has been deployed in multiple studies. Neural networks look for dependencies in the data, as opposed to simulation models, which are based on interaction rules between objects defined initially. Malavasi and Ricci [28] use neural networks to predict the total experienced delay on a railway line, given its geometrical and technological characteristics, and its scheduled utilization over time. In comparison to simulation, Malavasi and Ricci find neural networks more robust against extreme-valued input, which implicates more likely case-overfitting with simulation. Kecman et al. [29] propose a Bayesian network delay prediction model. In this case, input includes the timetable and recorded delays at all stations. Each delay is assumed to depend only on direct connections in a timed event graph, meaning the recorded delay for the same train at a previous station and for the previous train at the same station. Conditional delay distributions are assumed to be Gaussian, and the parameters 
are derived through recursive Generalized Linear Models. Chapuis [30] deploys the same assumed delay dependency in a neural network model, where input includes delay of the previous train and at the previous station, and distance to the next station. Such a model can predict the delay of a train at the next station. Independent of the actual infrastructure, this model is generic and can be applied at any station of the railway network. The downside of neural networks, though, is the risk of data overfitting, reducing the prediction capability, although this risk is lower in neural networks than in simulation models.

In response, Marković et al. [31] introduce Support Vector Regression (SVR) to establish a functional relationship between characteristics of the railway system and train delays. Train category, scheduled time, infrastructure, and share of journey completed are identified as most influencing factors to predict the train delay at one station. The authors show that SVR generalizes better than an artificial neural network, which seeks to minimize error of prediction in the historical dataset. Interestingly, the authors assume that the performance of delay prediction can be improved by grouping delays by magnitude, as factors generating smaller delays differ from factors that generate larger disturbances.

Kecman and Goverde [32] apply big data techniques to predict running and dwelling times from actual operation data, based on records from block sections occupations. The study uses random forests of tree-based models, to predict nonlinear relations between input variables and process times, with sufficient robustness to outliers in the data, lowered risk of overfitting, and focus on real time application. Running time predictors are calculated for every block section, and dwelling time predictors are calculated for every station platform. Among the interesting findings, the running times are longer if the headway to the preceding train is short, meaning that the succeeding trains tend to slow down to smoothen the trip and reduce the risk of encountering a yellow signal. Moreover, the authors find no evidence to support the hypothesis that trains run faster when delayed. All the trains were found to run at approximately the maximum performance in any condition. The authors suggest that, in case of insufficient prediction accuracy, new variables might be included in the model, such as platform shape for dwelling times.

Big data techniques focus mainly on the prediction of delays and running times or on the identification of delay chains and realized delay propagation among trains. New applications of these techniques would support the analysis of the realized development of delays along the path of individual train delays. As shown by statistical analysis and visual search for patterns presented by Schittenhelm and Richter [1, 18], Peterson [4], Andersson et al. [19, 21], and van Oort et al. [22], this type of data contains a great deal of information yet to be explored, which would provide insight into the effectiveness of running time supplements and into the presence of structural issues that generate delay in transport operation. In this paper we present a clustering technique to identify recurrent delay patterns among train services, based on readily available data, which leaves room for inference on the factors that generate specific delay patterns. The result shows that, within comparable train trajectories and stopping patterns, different train services accumulate delay at different stations and that recovery shapes differently according to the route direction. Inferences on the cluster composition show the most frequent service characteristics in each cluster. Such information could inform the allocation of correction measures to improve timetables. Tables 1 and 2 summarize the literature just reviewed.

\section{Identification of Recurrent Delay Patterns Using Big Data Techniques}

In this paper, a delay profile of a train run is defined as the set of recorded deviations throughout its path or a part of it, on a specific date. Note that deviation is reported as the time difference between a scheduled and a realized event, such as arrival, departure, or nonstop timing point. Even though delay is often used to refer to positive deviations, a delay profile can include null and negative values. A delay profile is a powerful representation of operation and the comparison of several delay profiles along the same service path allows the identification of recurrent delay patterns and such a representation method has already been presented in the literature $[1,4,18,19,21,22]$. Delay change, also called delay jump, is the difference in deviation between two consecutive stations and represents the delay recovery or increase. Schittenhelm and Richter [1] use this measure to assess delay increases or time gains between stations, and Goverde and Meng [11] use it to identify delay chains in railway operation. We define a delay change profile of a train as the set of recorded delay changes along its path or a part of it.

A dataset of delay profiles consists of all the delay profiles recorded in a defined period, stacked together. Fields, or variables, of the dataset are the events at every station, whereas observations are individual train runs from a selected service. Such a dataset can refer to a specific train service or to several services following the same stopping pattern, so that the fields can be aggregated. The first case is intended for infrequent services, typically long-distance trains, where every single service may have its own characteristics in terms of planned demand, scheduled rolling stock, or the time of crossing congested nodes. Suburban and regional railway services are often scheduled in constant stopping patterns at high frequency and could, thus, be analyzed together, expecting characteristics of operation to be more homogenous across services. A dataset of delay change profiles is defined analogously to delay profile datasets, where the fields contain the change in deviation in place of the absolute deviation.

Previous researches presented on delay and delay change profiles interpret recurrent patterns by visual search for similarities $[1,4,19,21]$. The systematic analysis of these two types of datasets through clustering algorithms allows the identification of patterns that are not necessarily visible or that could be wrongly associated by subjective interpretation.

Clustering techniques partition a dataset into a collection of groups of similar observations. In this study, clustering is used to partition the datasets of delay profiles and 


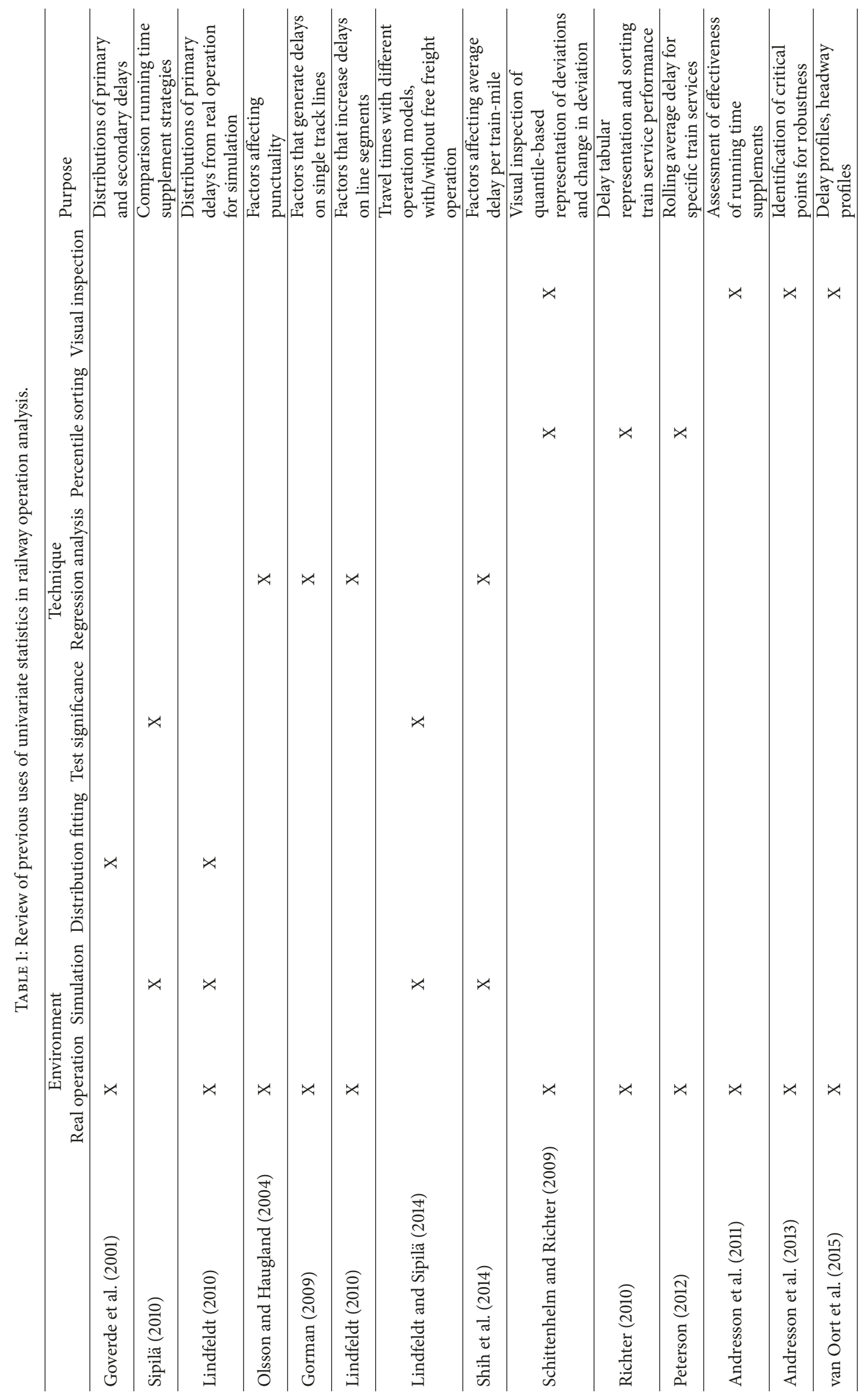




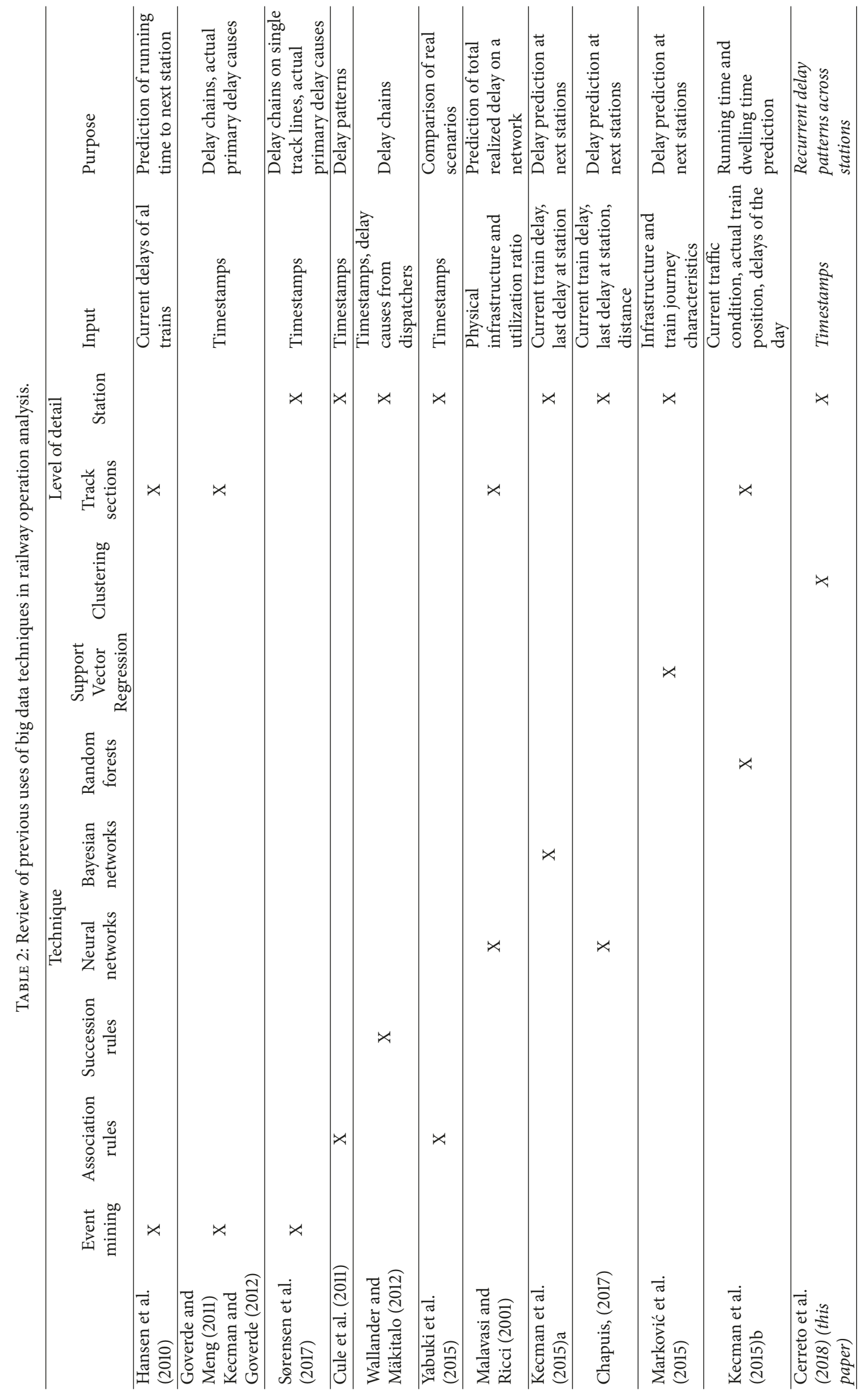


identify train services that are candidates for identification of common causality. Inference on common factors appearing in observations clustered together facilitates the assessment of delay patterns in association with specific characteristics of a transport service, such as time of the day (peak/offpeak), day of the week, or equipment used. The clustering process is realized through measures of similarity between elements in the same cluster and dissimilarity between elements from different clusters. Several methods and metrics are available to accomplish the task, suitable for different uses. Hierarchical algorithms proceed by splitting or merging observations recursively and are preferred when a nested structure is assumed in the clusters. In contrast, partitional algorithms do not impose a hierarchical structure and find all the clusters at the same time. $K$-means clustering is a partitional algorithm and was chosen due to its simplicity and frequent appearance in the literature [33].

$K$-means clustering is an iterative clustering process based on the identification of the mean element in each cluster. Every cluster is represented by its centroid, calculated as the average of the elements of the cluster, and every observation is assigned to the cluster corresponding to the closest centroid. Given a number $k$ of initial centroids, the algorithm executes the following steps:

(1) Assigning every element to the cluster with the closest centroid

(2) Calculating the new centroids of all the clusters as the mean of the elements

(3) Repeating until convergence, which is met when no element changes cluster between consecutive iterations.

This simple method requires three user-specified parameters, which might be hard to determine beforehand: the distance metric, the number of clusters $k$, and the cluster initialization. Euclidean distance is often used to determine the difference between observations, but other metrics are available, such as the $L_{1}$ distance [34]. The number of clusters $k$ is the most difficult parameter to estimate, as there is no perfect mathematical criterion. The parameter $k$ is typically determined according to available knowledge about the data or interpreting and evaluating the meaning of several independent partitions realized for different values of $k$. The initial centroids might influence the resulting clusters, so the initialization is often chosen among several independent partitions that result from sampling $k$ initial centroids among the observations. The influence of initialization, however, generally diminishes with the dimensionality of the dataset [33].

A substantial contribution to the simplicity of the method is given by the required structure of the data. Contrary to observer-operated search, clustering methods rely on the numerical relations between variable values recorded across single observation. It is, thus, unnecessary for the clustering algorithm to preprocess the data and sort the recorded delays for every train/observation. In the method proposed in this paper, $k$-means clustering is applied to observations of a multidimensional variable, whose size corresponds to the number of timing points of a fixed stopping pattern, where

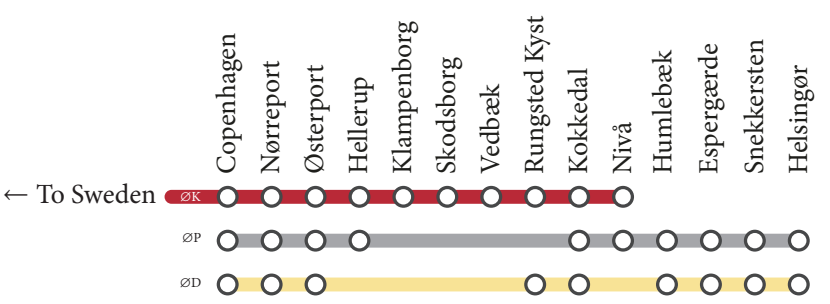

FIGURE 1: DSB services and stopping patterns on the Kystbanen.

the fields contain the delays, or delay changes, respectively, recorded at the individual timing points. Every observation of this multidimensional variable is a vector and represents a single train run.

\section{Case Study: The Kystbanen, Copenhagen}

The Kystbanen (coastline) is a double-tracked railway in the Copenhagen region. It is one of the busiest railway lines in the network of Banedanmark, the Danish infrastructure manager, and it is operated with regional standards, with some international services. It is operated nearly entirely by DSB, the largest Danish railway undertaking, which runs three different service types. The timetable is cyclic, and the services operate different stopping patterns during the day, as illustrated in Figure 1.

(i) The Øresund trains (“ØK") run all day every 20 minutes on a limited section of the coastline, between Copenhagen and Nivå. These trains operate between Denmark and Sweden across the Øresund bridge and stop at every station in Danish territory.

(ii) The regional trains ("ØР") run all day every 20 minutes as well, but they only operate in Denmark and run the whole coastline. These trains skip selected stops between Copenhagen and Nivå.

(iii) Additional trains are operated in the morning and afternoon peak hours. The rush hour trains ("ØD") operate every 20 minutes between Copenhagen and Helsingør, skipping other selected stops.

Fewer trains with modified stopping patterns run at night, so only weekday operation between $4: 30$ and 20:00 is considered in this study. The sections between Copenhagen and Østerport and between Snekkersten and Helsingør are shared with other services and operators.

In the resulting charts, stations are identified by a code specified by the infrastructure manager. Station codes and names are reported in Table 3.

Banedanmark provided a set of timestamps that state the scheduled and realized times of the trains at every timing point from April to December 2014. The records include information about the operation and about the timing points, such as station name, train ID, train category, scheduled time, and recorded deviation. Banedanmark relies on automatic train detection systems, based on the signaling system components. Typically, the track circuit boundaries do not correspond exactly to the platforms, and an offset 
TABLE 3: Station codes and names on the Kystbanen.

\begin{tabular}{lcc}
\hline Station code & Station name & $\begin{array}{c}\text { Distance from KH } \\
{[\mathrm{km}]}\end{array}$ \\
\hline KH & København H & 0,0 \\
KN & (Copenhagen central station) & 1,5 \\
KK & Nørreport & 3,1 \\
HL & Østerport & 7,8 \\
KL & Hellerup & 13,3 \\
SA & Klampenborg & 18,8 \\
VB & Skodsborg & 22,1 \\
RU & Vedbæk & 26,1 \\
OK & Rungsted Kyst & 29,1 \\
NI & Kokkedal & 32,5 \\
HUM & Niva & 36,3 \\
GÆ & Humlebæk & 40,0 \\
SQ & Espergærde & 42,7 \\
HG & Snekkersten & 46,2 \\
\hline
\end{tabular}

is generated between the time recorded by the automatic system and the actual time a train arrives at the platform or departs. This is a rather common problem, and it is also reported in the Netherlands [32] and Norway [12]. For the Danish network, a correction factor was calculated by Banedanmark using statistical analyses of GPS positions of train trajectories in collaboration with the main rail operator, DSB. The method and results are described by Richter et al. $[35,36]$. Nørreport station is the only underground station on the line, so GPS correction is not available, which is visible as a sawtooth pattern common to all train services in the delay profiles presented below, with a slightly underestimated delay for arrival records at Nørreport and overestimated one for departure records from the same station. Similarly, delay change records are shifted to negative values for arrivals at Nørreport and at Østerport, whereas higher positive values are recorded for delay changes at departures from Nørreport. The bias is systematic and has the same exact effect on all the trains; therefore its influence on clustering can be neglected.

The train timestamps were rearranged by an automatic algorithm to create datasets as described in Section 3, by means of the commercial software SAS 9.4 TS Level 1M4, by SAS Institute Inc., Cary, NC, USA. Observations corresponded to a realized train on a given date, and the fields contained the recorded delay at every station. Data from every station was divided into arrival, departure, and passthrough times, where trains did not stop. Each record is the delay profile or the delay change of a train on a date and represents one observation of the given train. Every variable identifies the station code and the type of timestamp, which can be entrance to the station, I ("Indkørsel"), exit from the station, U ("Udkørsel"), or pass-through station, G ("Gennemkørsel"), which is used where trains do not stop.

The analysis is intended to report delay patterns. Consequently, punctual trains are discarded from the dataset. In
Denmark, punctuality measurements are based on a delay threshold of 5 minutes for regional and long-distance trains, such as the Kystbanen. However, for internal management purposes, the infrastructure manager Banedanmark creates a delay report every time a train reaches at least 3 minutes of delay, containing information on the delay cause and on possible other trains hindered. Consequently, only trains with at least one recorded delay greater than or equal to 3 minutes are considered relevant in the present case study. Delay distributions are known to include large shares of trains with short delays, with decreasing frequency for larger delays $[7,8]$. Largely unbalanced clusters are a known issue in clustering algorithms and are an object of study to reduce the interference of large clusters [37]. In this case, punctual trains can therefore be considered as a compact cluster derived by prior knowledge, and they can be filtered out from the cluster analysis. The operation of filtering can be considered noise reduction and improves the quality of clustering, as the $k$-means procedure tends to generate spherical clusters of same radius [38]. According to Marković et al. [31], large delays are influenced by different factors other than smaller delays, which further supports the filtering choice. However, in different contexts, the filtering threshold might be set equal to a different value or not be applied at all.

Given the characteristic high frequency of train services on this line, clustering was operated by stopping patterns rather than by train numbers, so trains were grouped together by direction and service category. Grouping trains with similar characteristics and same stopping patterns increases data availability in the comparison and does not disqualify the result. In fact, such grouping was already proposed by Schittenhelm and Richter [1].

As explained in Section 3, $k$-means clustering requires choosing the number of clusters $k$ in advance. To set the number of clusters, the $k$-means algorithm was repeated with different values of $k$, and the best result was selected using criteria from Jain [33]. The number of clusters $k$ should be large enough to represent different patterns. At the same time, as $k$ increases, the same delay patterns tend to split into more clusters, and $k$ should remain small enough to prevent the generation of duplicate clusters. In detail, for every combination of train category, direction, and clustering variable (delay or delay change), $k$ was set as the highest integer that did not generate duplicate clusters. That is, the univariate distributions of delays, or delay changes, in every cluster should be different from all the other clusters for at least one station. Since $k$ is selected independently for all the mentioned cases, the same set of trains might best be represented by a different number of clusters when the algorithm operates on the delay variables or on the delay change variables. The $L 1$ distance was used as a clustering metric between observations, as suggested by Kashima et al. [34].

$K$-means clustering was performed on the described dataset by the commercial software MATLAB R2017a, by The MathWorks, Inc. Selected results of the method application are reported in Section 4.1, clustering on either delay profiles or delay change profiles. 

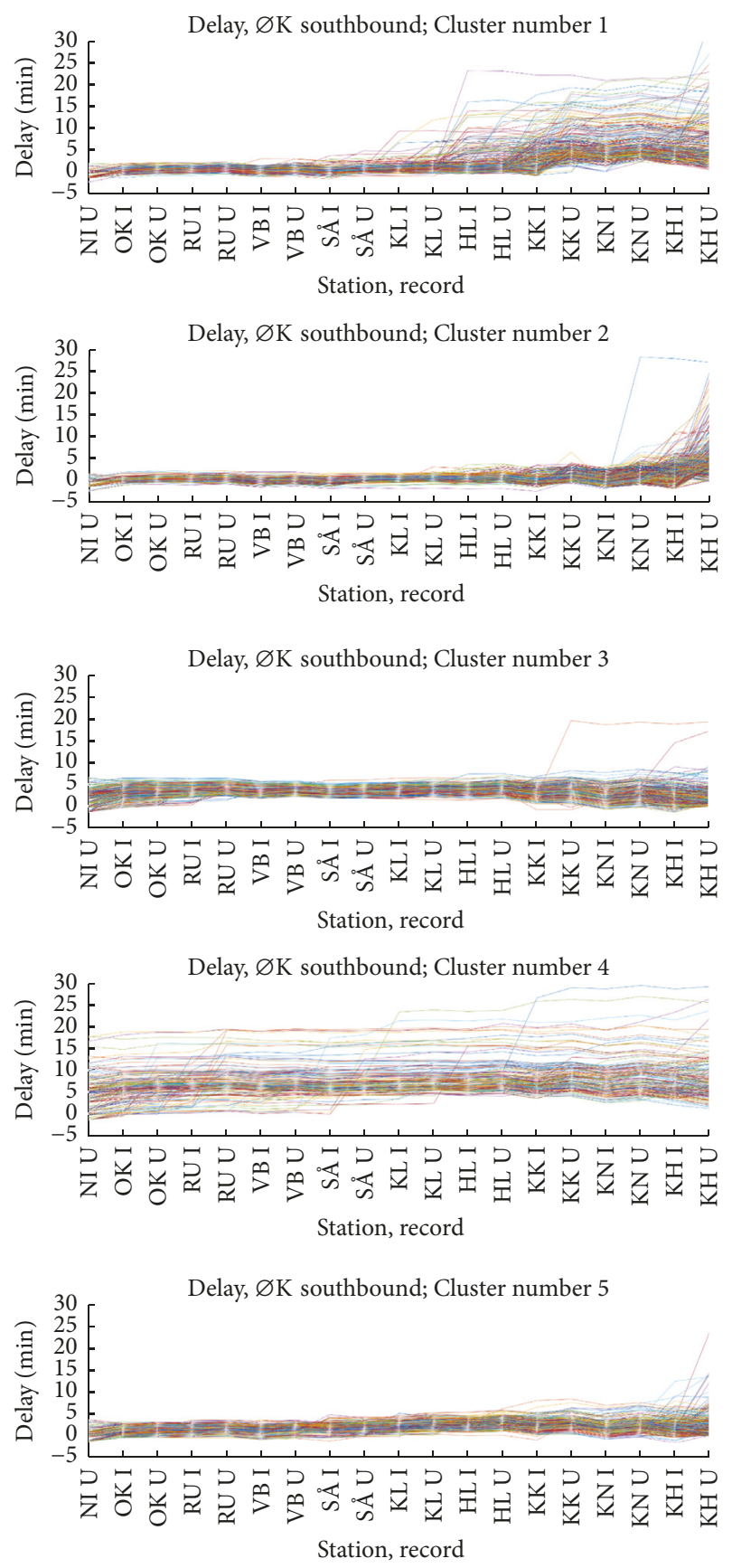

FIgURE 2: Resulting clusters in southbound ØK trains, Nivå-Copenhagen.

4.1. Clustering Results. Figure 2 illustrates the effectiveness of delay profiles clustering on $\varnothing \mathrm{K}$ southbound trains, on the delay variables. Note, after a stop at Copenhagen central station, these trains proceed to Sweden. The charts show that similar delay profiles are grouped together with low variance around the average centroid of each cluster, highlighting recurrent patterns. The resulting clusters can be interpreted as follows:

(1) Cluster 1: it includes trains that are punctual on the first section of the line but suffer delays approaching the most congested area of Copenhagen, mainly from Klampenborg and from Østerport.

(2) Cluster 2: it includes trains that are punctual throughout the complete journey, which receive delays leaving from Copenhagen.

(3) Cluster 3: it includes trains that are nearly punctual or anyway within 5 minutes of delay through the complete journey and across Copenhagen central station; from Hellerup, a marginal delay recovery is visible for these trains.

(4) Cluster 4: it includes the most delayed trains, being delayed throughout the whole itinerary or it largest part.

(5) Cluster 5: it includes punctual trains with slightly, but steadily, increasing delay across stations.

Some clusters present outliers, such as Clusters 2 and 3. Even though some delay profiles may appear considerably different from other profiles in the same clusters, these observations were assigned to the cluster with the closest centroid. This means that, in selected cases, the delay profiles are the representation of rather unique events, which may be neglected after more detailed analysis in the composition of the individual clusters.

Individual clusters are characterized through the mean values of the aforementioned measures. The following measures were computed for each train run to characterize the individual clusters:

(i) Average, minimum, and maximum delay across stations

(ii) Range of delays across stations

(iii) Standard deviation of delays recorded across stations

(iv) Initial delay, the delay at first station

(v) Final delay, the delay at the last station

(vi) Overall delay change, difference between final and initial delay-positive values mean the delay has increased from first to last station

(vii) Maximum delay change across stations.

Cluster characteristics are summarized in Table 4.

4.2. Comparison with Percentile-Based Approaches on Delay Profiles. In this section, a comparison is provided between the pooled data and the clusters on the dataset of delay profiles. The same percentile representation of delay profiles is shown, as proposed by Schittenhelm and Richter [1], Peterson [4], and van Oort et al. [22]. These authors represented different percentiles. For the sake of clarity, only the 15th, 50th, and 85th percentiles and the average are displayed in the following diagrams.

Figure 3 shows the distribution of delays of the entire dataset of $\varnothing \mathrm{K}$ southbound trains. The only pattern visible is a slight increase in delay towards Copenhagen, more evident for the more delayed trains, represented by the 85 th percentile. Even though a large portion of punctual trains 
TABLE 4: Characterization of delay profile clusters and southbound ØK trains, Nivå-Copenhagen.

\begin{tabular}{lcccccccccc}
\hline Cluster & $\begin{array}{c}\text { Number of } \\
\text { observations }\end{array}$ & $\begin{array}{c}\text { Mean } \\
\text { average } \\
\text { delay } \\
\text { [min] }\end{array}$ & $\begin{array}{c}\text { Mean } \\
\text { STD of } \\
\text { delays } \\
{[\mathrm{min}]}\end{array}$ & $\begin{array}{c}\text { Mean } \\
\text { initial } \\
\text { delay } \\
\text { [min] }\end{array}$ & $\begin{array}{c}\text { Mean final } \\
\text { delay [min] }\end{array}$ & $\begin{array}{c}\text { Mean min } \\
\text { delay [min] }\end{array}$ & $\begin{array}{c}\text { Mean max } \\
\text { delay [min] }\end{array}$ & $\begin{array}{c}\text { Mean delay } \\
\text { range [min] }\end{array}$ & $\begin{array}{c}\text { Mean max } \\
\text { delay change } \\
\text { [min] }\end{array}$ & $\begin{array}{c}\text { Mean overall } \\
\text { delay change } \\
\text { [min] }\end{array}$ \\
\hline$(1)$ & 270 & 2,26 & 2,78 & $-0,95$ & 6,14 & $-1,06$ & 7,72 & 8,78 & 4,88 \\
$(2)$ & 418 & 0,55 & 1,47 & $-1,05$ & 4,71 & $-1,24$ & 5,27 & 6,52 & 4,69 & 5,76 \\
$(3)$ & 381 & 3,09 & 1,12 & 1,70 & 1,80 & 0,53 & 4,64 & 4,11 & 2,69 & 0,11 \\
$(4)$ & 159 & 7,65 & 1,92 & 4,59 & 8,03 & 3,73 & 10,21 & 6,47 & 6,79 & 3,44 \\
$(5)$ & 395 & 1,92 & 1,14 & $-0,28$ & 2,23 & $-0,47$ & 4,10 & 4,57 & 2,25 & 2,51 \\
\hline Total & 1623 & 2,46 & 1,57 & 0,35 & 3,99 & $-0,12$ & 5,73 & 5,85 & 3,87 \\
\hline
\end{tabular}

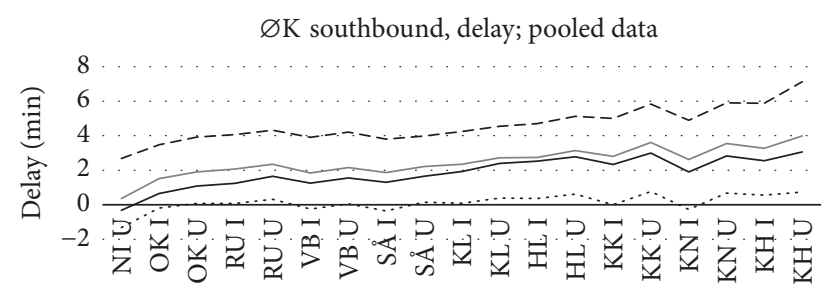

FIGURE 3: Delays recorded for $\emptyset \mathrm{K}$ southbound trains. 15th percentile: dotted; median: solid black; 85 th percentile: dashed; average: solid gray.

was discarded from the dataset, the residual distribution of delays remains positively skewed, as shown by the average constantly higher than the median value.

The new information revealed by the clustering algorithm is provided in Figure 4. In this figure, the individual internal distributions of delays are compared to the pooled delay distribution from Figure 3. Figure 4 shows, for each cluster, the difference between the cluster statistic at each station and the equivalent pooled statistic from Figure 3.

In Figure 4, the 15th and 85th percentiles and the median line of the internal cluster delay profiles distributions are compared to the distribution of pooled delay profiles. The clusters where the difference of 85 th percentile from the pooled dataset is lower than the difference of the 15th percentile have tighter distributions of delay profiles compared to the pooled dataset, increasing the significance of the identified pattern. The local deviation present in the clusters represents the information hidden in the pooled dataset, which is instead brought to light by the clustering.

\subsection{Comparison with Percentile-Based Approaches on Delay} Change Profiles. In this section, a comparison is provided between the pooled data and the clusters on the dataset of delay change profiles. The same representation of delay change profiles based on the median is shown, as proposed by Schittenhelm and Richter $[1,18]$, supplemented with the average, that is, the cluster centroid.

Figure 5 shows the delay change profiles of the entire dataset of $\varnothing \mathrm{D}$ northbound trains. A generalized positive delay change is visible at the last station. The large changes in delay from location KN I to KK I are linked to the known deviation in the timestamps at Nørreport.

The differences between the pooled median and average delay change profile and the same profiles from individual clusters are represented in Figure 6. In this case, the information gained by clustering is more evident. All the clusters remain similar to the pooled data at most stations, except few stations, where a large difference is recorded in the delay change.

Every cluster is characterized by at least one larger delay change at one station, which would be hidden in the pooled distribution of delay change profiles. Noticeably, the negative effect of different delay patterns overlapping is evident for $K N$ $I$ records. All the clusters deviate negatively from the pooled data by around 0,5 minutes, except Cluster 2, which deviates positively by around 1,5 minutes from the pooled profile. This means that the pooled profile was shifted by one single cluster to a central value, hiding both the frequent delay recovery and the specific delay increase from Cluster 2.

4.4. Inference on the Clusters. In this section, results from clustering of delay profiles and delay change profiles are investigated to identify relations with cluster characteristics, using heuristic classification. For the sake of conciseness, only cluster centroids are reported in Figures 7 and 10, and only a sample of the results is reported, which is $\varnothing \mathrm{D}$ northbound trains and $\varnothing \mathrm{K}$ southbound trains. Figure 7 shows results from clustering delay change profiles for $Ø \mathrm{D}$ trains to Helsingør.

Clusters can be interpreted as follows:

(1) Cluster 1: regular delay increases at the last three stations, where trains become unpunctual.

(2) Cluster 2: delay increase arriving at the first stop, Nørreport.

(3) Cluster 3: trains are considerably delayed arriving at the final destination, Helsingør.

(4) Cluster 4: trains are without remarkable delay changes: these trains tend to keep the same delay throughout the whole journey.

(5) Cluster 5: specific delay increases at Humlebæk arrival; trains in this cluster show also smaller recovery at Skodsborg arrival, compared to other clusters. 

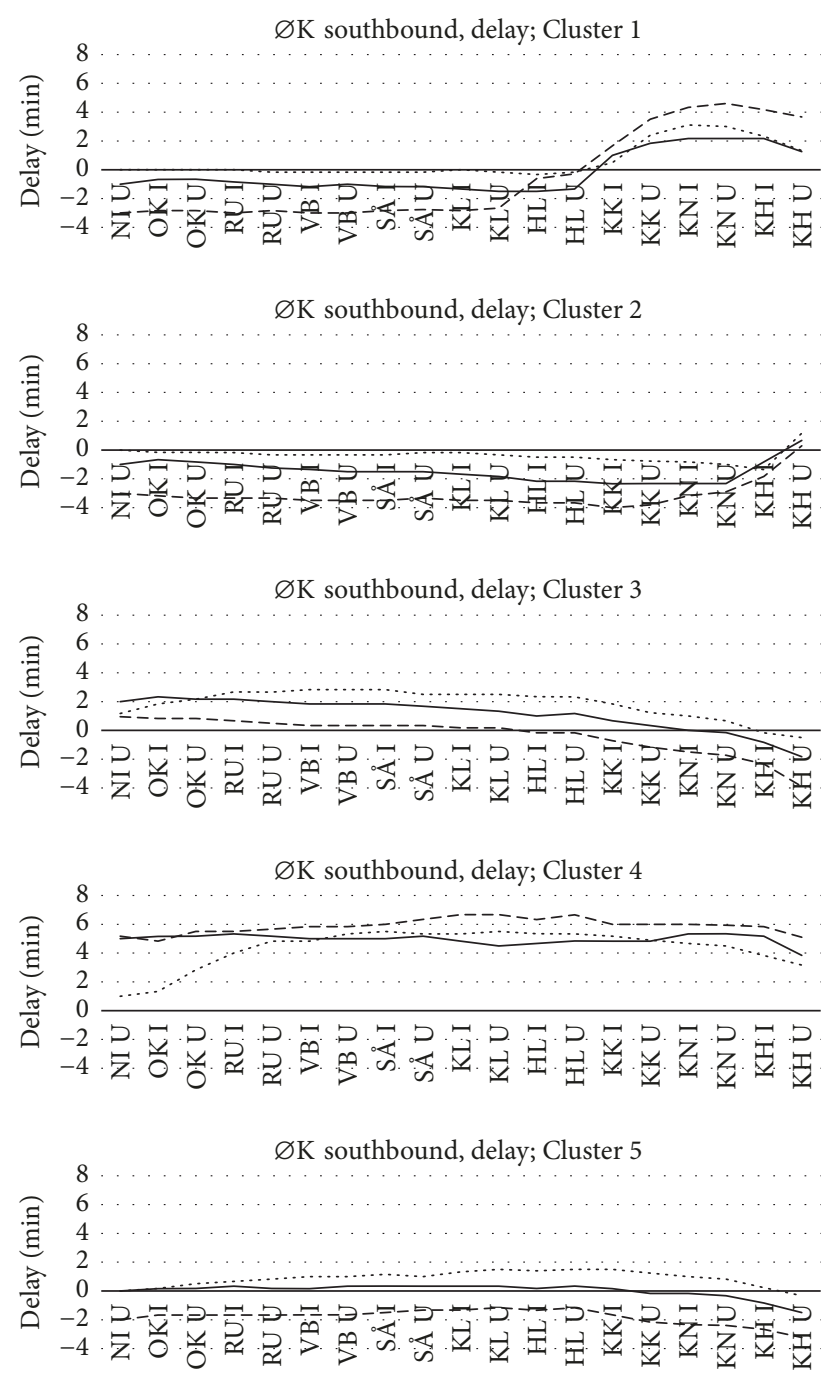

FIGURE 4: Differences in the distributions of delays recorded for $\emptyset \mathrm{K}$ southbound trains. Each cluster's internal distribution is compared to the pooled distribution. 15th percentile: dotted; median: solid; and 85th percentile: dashed.

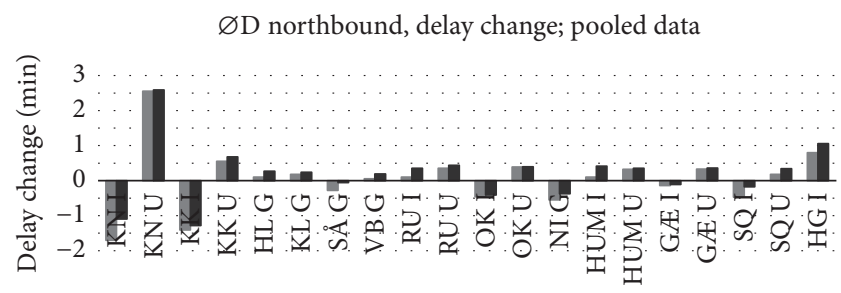

FIGURE 5: Delay changes recorded for $\varnothing D$ northbound trains. Median in bright shade and average in dark shade.

(6) Cluster 6: these trains accumulate delays passing the stations of Hellerup and Klampenborg; on the other side, compared to other clusters, the average delay increase at final destination Helsingør is smaller.

Inference on the cluster population shows that some patterns are specific for selected train services, identified by their
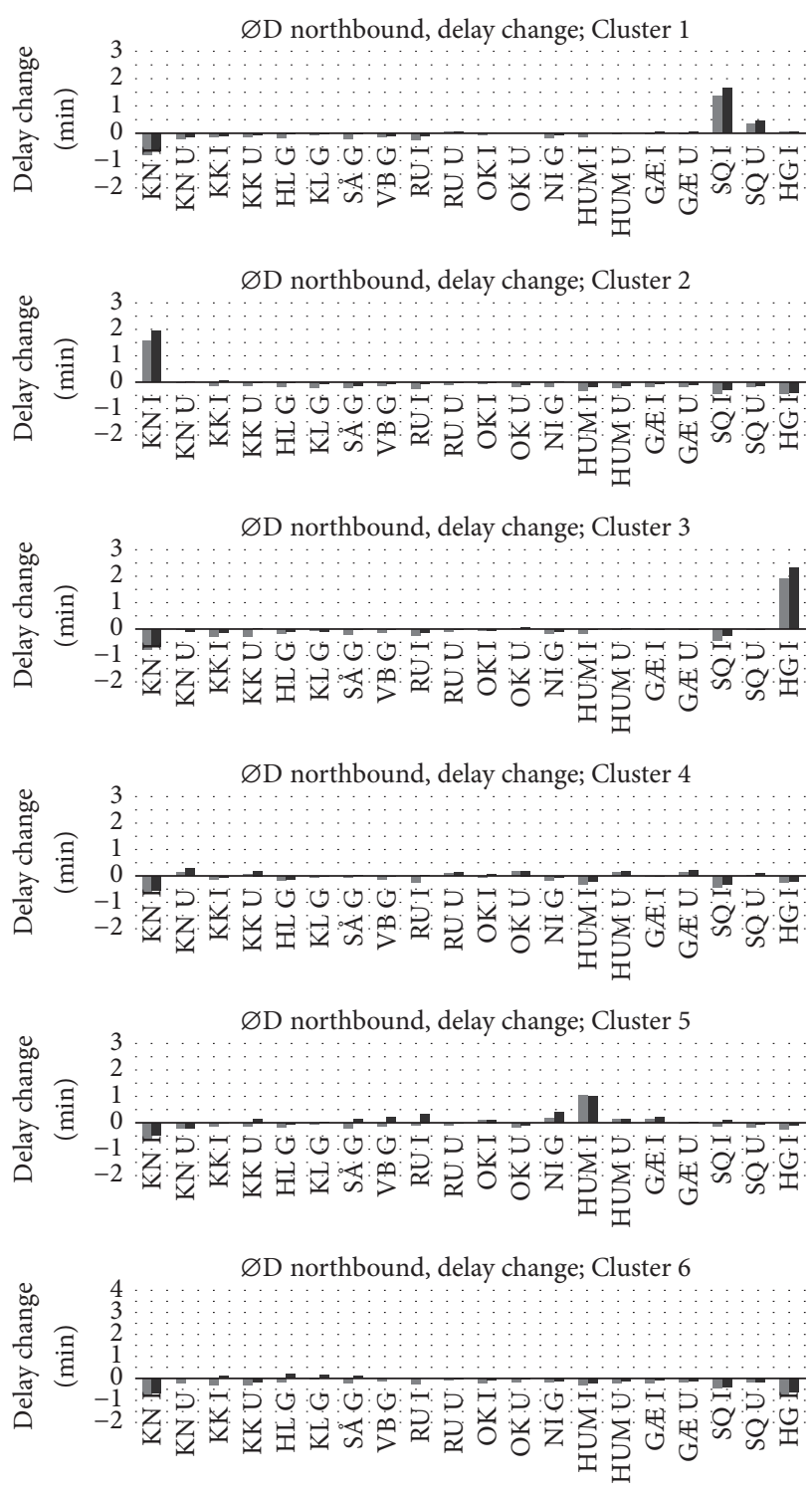

FIGURE 6: Delay changes recorded for $\varnothing \mathrm{D}$ northbound train, by clusters. Median in bright shade and average in dark shade.

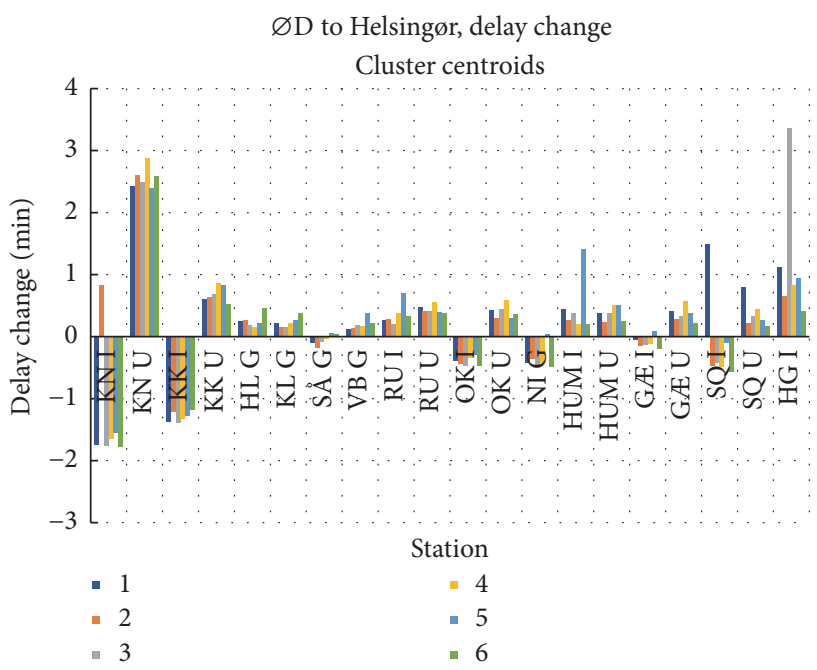

FIGURE 7: Cluster centroids for northbound ØD trains: delay change. 


\begin{tabular}{|c|c|c|c|c|c|c|c|c|}
\hline \multirow{2}{*}{$\begin{array}{l}\text { Time } \\
\text { band }\end{array}$} & \multirow{2}{*}{$\begin{array}{c}\text { Departure } \\
\text { time from } \\
\mathrm{KH} \\
\end{array}$} & \multirow{2}{*}{$\begin{array}{l}\text { Train } \\
\text { number }\end{array}$} & \multicolumn{6}{|c|}{ Cluster } \\
\hline & & & 2 & 6 & 4 & 1 & 3 & 5 \\
\hline \multirow{4}{*}{ 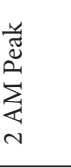 } & $06: 18$ & 4413 & $18 \%$ & $25 \%$ & $14 \%$ & $14 \%$ & $18 \%$ & $11 \%$ \\
\hline & $06: 38$ & 4415 & $4 \%$ & $36 \%$ & $4 \%$ & $16 \%$ & $32 \%$ & $8 \%$ \\
\hline & $06: 58$ & 4417 & $26 \%$ & $33 \%$ & $7 \%$ & $19 \%$ & $4 \%$ & $11 \%$ \\
\hline & 07:18 & 4419 & $6 \%$ & $22 \%$ & $8 \%$ & $31 \%$ & $8 \%$ & $25 \%$ \\
\hline \multirow{10}{*}{ 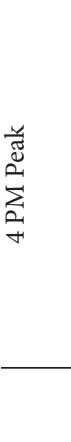 } & $15: 18$ & 4467 & $21 \%$ & $25 \%$ & $17 \%$ & $4 \%$ & $21 \%$ & $13 \%$ \\
\hline & $15: 38$ & 4469 & $19 \%$ & $30 \%$ & $7 \%$ & $12 \%$ & $23 \%$ & $9 \%$ \\
\hline & $15: 58$ & 4471 & $44 \%$ & $16 \%$ & $16 \%$ & $4 \%$ & $8 \%$ & $12 \%$ \\
\hline & $16: 18$ & 4473 & $5 \%$ & $15 \%$ & $28 \%$ & $18 \%$ & $20 \%$ & $15 \%$ \\
\hline & $16: 38$ & 4475 & $43 \%$ & $13 \%$ & $21 \%$ & $13 \%$ & $10 \%$ & $2 \%$ \\
\hline & $16: 58$ & 4477 & $20 \%$ & $15 \%$ & $39 \%$ & $9 \%$ & $2 \%$ & $15 \%$ \\
\hline & $17: 18$ & 4479 & $16 \%$ & $32 \%$ & $15 \%$ & $12 \%$ & $9 \%$ & $16 \%$ \\
\hline & $17: 38$ & 4481 & $31 \%$ & $14 \%$ & $19 \%$ & $19 \%$ & $5 \%$ & $12 \%$ \\
\hline & $17: 58$ & 4483 & $46 \%$ & $14 \%$ & $14 \%$ & $6 \%$ & $10 \%$ & $10 \%$ \\
\hline & & $\begin{array}{c}\text { Cluster } \\
\text { share }\end{array}$ & $24 \%$ & $22 \%$ & $17 \%$ & $14 \%$ & $12 \%$ & $12 \%$ \\
\hline
\end{tabular}

FIGURE 8: Northbound ØD trains. Cluster share by train service ID. The color code compares the individual row's distributions among clusters to the overall distribution among clusters reported in the last row. Clusters sorted by size.

\begin{tabular}{|c|c|c|c|c|c|c|c|c|}
\hline \multirow{2}{*}{ Time } & \multirow{2}{*}{ Type } & \multirow{2}{*}{ Time band } & \multicolumn{6}{|c|}{ Cluster } \\
\hline & & & 2 & 6 & 4 & 1 & 3 & 5 \\
\hline $6: 20-8: 20$ & Peak AM & 2 & $13 \%$ & $28 \%$ & $9 \%$ & $21 \%$ & $15 \%$ & $15 \%$ \\
\hline \multirow[t]{2}{*}{$15: 20-18: 00$} & Peak PM & 4 & $27 \%$ & $20 \%$ & $19 \%$ & $12 \%$ & $11 \%$ & $11 \%$ \\
\hline & & $\begin{array}{l}\text { Cluster } \\
\text { share }\end{array}$ & $24 \%$ & $22 \%$ & $17 \%$ & $14 \%$ & $12 \%$ & $12 \%$ \\
\hline
\end{tabular}

Figure 9: Northbound ØD trains. Cluster share by time band. The color code compares the individual row's distributions among clusters to the overall distribution among clusters reported in the last row. Clusters sorted by size.

train number. Figure 8 shows how every train service ID is spread across clusters. In each column, the shade represents the difference between individual percentages and the cluster share, where the brightest colors are associated with the values furthest from the cluster share. Green is positive difference, that is, larger percentages than the cluster share; red is negative difference, that is, smaller percentages than the cluster share.

Delay change profiles in Clusters 1 and 5 represent typical behavior of service 4419, whereas Cluster 2 is considerably more frequent in services 4471, 4473, and 4483. Cluster 3 is more common among services 4415 , three times more frequent than the whole population distribution across clusters, 4467,4469 , and 4473, which double the average frequencies. Cluster 4 is typical for service 4477 , and, lastly, Cluster 6 represents a large share of services 4417 and, again, 4415. Further investigation on other factors may reveal the causes that rule the train services' cluster membership.

The analysis of Figure 8 shows the existence of a relation between train IDs in a specific time band and cluster membership. This is shown in detail in Figure 9, where cluster membership is aggregated in time bands. The same color coding as Figure 8 is applied.

The timetable is divided into time bands according to the overall service frequency on the line, so that time bands 2 and 4 are the AM and PM peak periods, respectively, when 9 trains/h per direction are operated. Time bands 1, 3, and 5 are the remaining off-peak periods, when $\varnothing \mathrm{D}$ trains are not operated, so only 6 trains/h occupy the line in each direction, allowing for larger headway buffers between trains. At the same time, smaller congestion is expected, in offpeak periods, both on the train traffic and on the number of passengers to board or alight at the stations.

In this case, morning peak shows recurrent delay patterns presented by Clusters 1 and 6, whereas patterns represented by Clusters 2 and 4 are rare in this time band. Oppositely, the distribution of trains in the PM peak hour is similar to the overall distribution.

Further inference on the clusters of $\varnothing \mathrm{D}$ northbound trains might highlight interferences from other trains. Lokaltog trains run mostly on a network independent of Banedanmark's and share with $\varnothing \mathrm{D}$ and $\varnothing \mathrm{P}$ trains the line section between Snekkersten and Helsingør. ØD northbound trains are scheduled at a short headway after Lokaltog trains from Snekkersten to Helsingør. The analysis of timestamps from Lokaltog trains on this section and of the realized headways between Lokaltog and $\varnothing \mathrm{D}$ northbound trains might suggest that Clusters 1 and 3, which increase the delay near Helsingør, are actually the result of delay propagation from Lokaltog trains to ØD trains. 


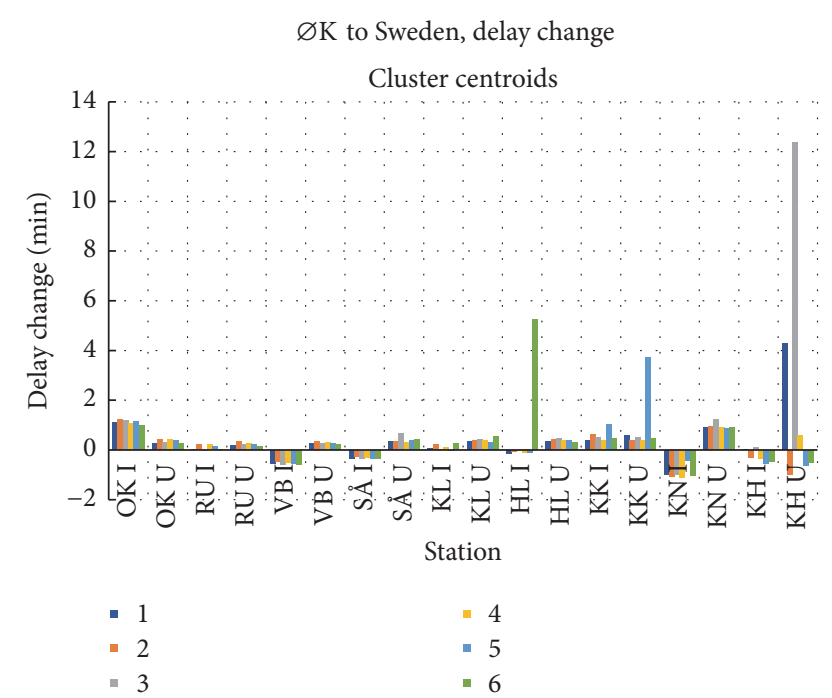

FIGURE 10: Cluster centroids for southbound $\varnothing \mathrm{K}$ trains: delay change.

The clustering results from other service categories, with different stopping patterns, can be related to the time periods of the day. For example, $\varnothing \mathrm{K}$ southbound trains are reported in Figures 10 and 11.

Figure 10 represents the centroids of resulting clusters in train category $\varnothing \mathrm{K}$ southbound, according to delay change. Besides, the distribution of trains across clusters is summarized in Figure 11, disaggregated by time bands, highlighted in the same color code as Figures 8 and 9. Note that the number of clusters in the delay change profiles of $\varnothing \mathrm{K}$ southbound trains is different from the number of clusters in delay profiles. This is not necessarily inconsistent, as the two variables express different aspects of the development of delays. In this case, the cluster share by time band explains the nature of two clusters. In particular, Cluster 1 is considerably more frequent in the PM peak hour, whereas Cluster 2 is more typical of trains in the AM peak hour. This result can be reasonably interpreted as delays generated by passenger congestion. In fact, delay increases in the PM peak hour appear at departures from Copenhagen, where a large number of passengers leave towards Sweden. On the contrary, Cluster 2 represents delays increases collected across stations towards Copenhagen and a delay recovery departing from Copenhagen, where fewer passengers are expected to board. The cluster share for Clusters 3 to 6 is comparable with the overall distribution across different time bands, so these delay patterns cannot directly be associated with time of the day. Further research may reveal factors that rule the cluster membership for these clusters.

More disaggregated analysis of cluster composition according to train number, or service ID, is in accordance with aggregated time bands. This is valuable especially for time band 3, which is the most populated time band according to the timetable. Figure 12 shows that even if the overall distribution of trains in time band 3 across clusters is very similar to the overall distribution, specific train services present different typical delay patterns. In this case, further analysis of train service characteristics should indicate a better disaggregation of train services in a specific time band. The same color code as in tables in Figures 8, 9, and 11 is applied in Figure 12.

Even though recurrent patterns are also clear in the delay profiles dataset, the results could not be explained by the available variables. Further research might identify relations that guide the clustering of delay profiles on this line, such as realized headways, weather conditions, passenger counts, and recorded delay causes.

\section{Discussion}

The clustering method proposed in this paper finds it strengths in being automatic, unbiased, flexible, and simple. A comparison to methods presented in the literature is provided in this section. Previous approaches $[1,4,18,19,21,22]$ extracted information from delay profiles mainly through observation, occasionally combined with multiple univariate statistical analyses and observation ranking. In most studies, the complete dataset was plotted in the form of delay profiles, and the identification of frequent patterns among the observations relied on the observer's ability. Visual inspection is typically affected by subjective interpretation, which can differ across analysts, and suffers from low effectiveness of naked eye to average data represented graphically. In some studies, supporting measures were plotted with the full dataset, such as average profile, median, and selected percentiles to represent the distributions.

The application of these measures as multiple univariate distributions, though, does not catch the interdependencies of delays at different stations and does not provide information about the development of delays along the train journey. The method proposed in this paper allows automatic identification of delay patterns, removing, thus, the influence of subjective interpretation of delay profiles. Furthermore, profile clustering allows the identification of similar delay profiles in the entire pool of records. Note that even though the clustered delay profiles were plotted in this paper, the observation of the profiles did not play a role in the identification of similarities. This exact process is indeed performed by the clustering algorithm, and the results are then plotted for an easier comprehension of the development of delays in the individual clusters. The metrics provided as 15 th, 50th, and 85 th percentile would be sufficient to describe the distributions within individual clusters and might be used in replacement of the cluster plots.

Compared to big data techniques proposed in the literature for other purposes in analysis of transport operation $[11,23,24,32]$, this method relies on readily available data and does not need detailed knowledge on the infrastructure and occupation of individual blocking sections. It can therefore be scaled to different levels of detail or transferred to other modes of transportation where delay can be measured at fixed points on a given path, such as bus networks or air traffic. It is a very common practice of transport operators to provide live data on delays recorded on their own network, which can be recorded accessing public websites. 


\begin{tabular}{lcccccccc}
\hline $\begin{array}{l}\text { Start } \\
\text { time }\end{array}$ & Type & Time band & \multicolumn{7}{c}{ Cluster } \\
\hline $04: 30$ & Off-peak & 1 & $35 \%$ & $30 \%$ & $15 \%$ & $12 \%$ & $3 \%$ & $5 \%$ \\
$06: 20$ & Peak AM & 2 & $49 \%$ & $26 \%$ & $10 \%$ & $11 \%$ & $1 \%$ & $3 \%$ \\
$08: 20$ & Off-peak & 3 & $39 \%$ & $29 \%$ & $13 \%$ & $13 \%$ & $3 \%$ & $3 \%$ \\
$15: 20$ & Peak PM & 4 & $30 \%$ & $26 \%$ & $24 \%$ & $9 \%$ & $9 \%$ & $2 \%$ \\
$18: 00$ & Off-peak & 5 & $40 \%$ & $29 \%$ & $18 \%$ & $9 \%$ & $3 \%$ & $1 \%$ \\
\hline & & Cluster share & $40 \%$ & $28 \%$ & $15 \%$ & $12 \%$ & $3 \%$ & $2 \%$ \\
\hline
\end{tabular}

FIGURE 11: Southbound $\varnothing \mathrm{K}$ trains. Cluster share by time band. The color code compares the individual row's distributions among clusters to the overall distribution among clusters reported in the last row. Clusters sorted by size.

\begin{tabular}{|c|c|c|c|c|c|c|c|c|}
\hline \multirow{2}{*}{$\begin{array}{l}\text { Time } \\
\text { band }\end{array}$} & \multirow{2}{*}{$\begin{array}{l}\text { Departure } \\
\text { time from NI }\end{array}$} & \multirow{2}{*}{$\begin{array}{c}\text { Train } \\
\text { number }\end{array}$} & \multicolumn{6}{|c|}{ Cluster } \\
\hline & & & 2 & 4 & 1 & 5 & 3 & 6 \\
\hline \multirow{3}{*}{1} & 06:01 & 1314 & $38 \%$ & $20 \%$ & $23 \%$ & $13 \%$ & $3 \%$ & $5 \%$ \\
\hline & $06: 21$ & 1316 & $32 \%$ & $44 \%$ & $8 \%$ & $8 \%$ & $4 \%$ & $4 \%$ \\
\hline & 06:41 & 1318 & $33 \%$ & $33 \%$ & $10 \%$ & $14 \%$ & $5 \%$ & $5 \%$ \\
\hline \multirow{6}{*}{2} & 07:01 & 1320 & $59 \%$ & $12 \%$ & $12 \%$ & $15 \%$ & $0 \%$ & $2 \%$ \\
\hline & 07:21 & 1322 & $50 \%$ & $29 \%$ & $13 \%$ & $4 \%$ & $0 \%$ & $4 \%$ \\
\hline & 07:41 & 1324 & $51 \%$ & $31 \%$ & $10 \%$ & $5 \%$ & $0 \%$ & $3 \%$ \\
\hline & 08:01 & 1326 & $41 \%$ & $32 \%$ & $14 \%$ & $11 \%$ & $2 \%$ & $2 \%$ \\
\hline & $08: 21$ & 1328 & $53 \%$ & $18 \%$ & $0 \%$ & $22 \%$ & $2 \%$ & $5 \%$ \\
\hline & 08:41 & 1330 & $42 \%$ & $33 \%$ & $14 \%$ & $3 \%$ & $3 \%$ & $6 \%$ \\
\hline \multirow{23}{*}{3} & 09:01 & 1332 & $43 \%$ & $30 \%$ & $4 \%$ & $20 \%$ & $0 \%$ & $4 \%$ \\
\hline & 09:21 & 1334 & $39 \%$ & $32 \%$ & $16 \%$ & $13 \%$ & $0 \%$ & $0 \%$ \\
\hline & 09:41 & 1336 & $41 \%$ & $24 \%$ & $15 \%$ & $17 \%$ & $0 \%$ & $2 \%$ \\
\hline & 10:01 & 1338 & $24 \%$ & $24 \%$ & $27 \%$ & $16 \%$ & $5 \%$ & $3 \%$ \\
\hline & $10: 21$ & 1340 & $31 \%$ & $39 \%$ & $19 \%$ & $3 \%$ & $0 \%$ & $8 \%$ \\
\hline & $10: 41$ & 1342 & $57 \%$ & $14 \%$ & $11 \%$ & $14 \%$ & $0 \%$ & $5 \%$ \\
\hline & 11:01 & 1344 & $45 \%$ & $24 \%$ & $12 \%$ & $15 \%$ & $0 \%$ & $3 \%$ \\
\hline & $11: 21$ & 1346 & $40 \%$ & $47 \%$ & $10 \%$ & $3 \%$ & $0 \%$ & $0 \%$ \\
\hline & 11:41 & 1348 & $53 \%$ & $35 \%$ & $7 \%$ & $5 \%$ & $0 \%$ & $0 \%$ \\
\hline & 12:01 & 1350 & $26 \%$ & $29 \%$ & $21 \%$ & $15 \%$ & $3 \%$ & $6 \%$ \\
\hline & $12: 21$ & 1352 & $47 \%$ & $32 \%$ & $8 \%$ & $8 \%$ & $0 \%$ & $5 \%$ \\
\hline & $12: 41$ & 1354 & $38 \%$ & $16 \%$ & $28 \%$ & $16 \%$ & $0 \%$ & $3 \%$ \\
\hline & 13:01 & 1356 & $46 \%$ & $19 \%$ & $15 \%$ & $8 \%$ & $8 \%$ & $4 \%$ \\
\hline & $13: 21$ & 1358 & $50 \%$ & $31 \%$ & $15 \%$ & $0 \%$ & $4 \%$ & $0 \%$ \\
\hline & 13:41 & 1360 & $59 \%$ & $24 \%$ & $7 \%$ & $3 \%$ & $7 \%$ & $0 \%$ \\
\hline & 14:01 & 1362 & $29 \%$ & $34 \%$ & $17 \%$ & $17 \%$ & $3 \%$ & $0 \%$ \\
\hline & $14: 21$ & 1364 & $44 \%$ & $32 \%$ & $12 \%$ & $4 \%$ & $8 \%$ & $0 \%$ \\
\hline & 14:41 & 1366 & $37 \%$ & $37 \%$ & $10 \%$ & $15 \%$ & $2 \%$ & $0 \%$ \\
\hline & 15:01 & 1368 & $24 \%$ & $24 \%$ & $16 \%$ & $30 \%$ & $4 \%$ & $1 \%$ \\
\hline & $15: 21$ & 1370 & $43 \%$ & $25 \%$ & $6 \%$ & $14 \%$ & $10 \%$ & $2 \%$ \\
\hline & $15: 41$ & 1372 & $35 \%$ & $41 \%$ & $5 \%$ & $11 \%$ & $5 \%$ & $3 \%$ \\
\hline & 16:01 & 1374 & $36 \%$ & $21 \%$ & $17 \%$ & $17 \%$ & $8 \%$ & $2 \%$ \\
\hline & $16: 21$ & 1376 & $36 \%$ & $33 \%$ & $14 \%$ & $6 \%$ & $6 \%$ & $6 \%$ \\
\hline \multirow{3}{*}{4} & $16: 41$ & 1378 & $37 \%$ & $27 \%$ & $22 \%$ & $7 \%$ & $5 \%$ & $2 \%$ \\
\hline & $17: 01$ & 1380 & $19 \%$ & $29 \%$ & $29 \%$ & $13 \%$ & $6 \%$ & $3 \%$ \\
\hline & $17: 21$ & 1382 & $39 \%$ & $18 \%$ & $18 \%$ & $5 \%$ & $18 \%$ & $0 \%$ \\
\hline \multirow{9}{*}{5} & $17: 41$ & 1384 & $45 \%$ & $29 \%$ & $23 \%$ & $3 \%$ & $0 \%$ & $0 \%$ \\
\hline & 18:01 & 1386 & $18 \%$ & $43 \%$ & $30 \%$ & $8 \%$ & $3 \%$ & $0 \%$ \\
\hline & 18:21 & 1388 & $47 \%$ & $28 \%$ & $16 \%$ & $9 \%$ & $0 \%$ & $0 \%$ \\
\hline & 18:41 & 1390 & $40 \%$ & $24 \%$ & $16 \%$ & $12 \%$ & $4 \%$ & $4 \%$ \\
\hline & 19:01 & 1392 & $58 \%$ & $15 \%$ & $13 \%$ & $15 \%$ & $0 \%$ & $0 \%$ \\
\hline & $19: 21$ & 1394 & $34 \%$ & $31 \%$ & $17 \%$ & $10 \%$ & $7 \%$ & $0 \%$ \\
\hline & 19:41 & 1396 & $48 \%$ & $26 \%$ & $7 \%$ & $7 \%$ & $11 \%$ & $0 \%$ \\
\hline & 20:01 & 1398 & $31 \%$ & $38 \%$ & $22 \%$ & $6 \%$ & $0 \%$ & $3 \%$ \\
\hline & & $\begin{array}{c}\text { Cluster } \\
\text { share }\end{array}$ & $40 \%$ & $28 \%$ & $15 \%$ & $12 \%$ & $3 \%$ & $2 \%$ \\
\hline
\end{tabular}

FIGURE 12: Southbound $\varnothing \mathrm{K}$ trains. Cluster share by service ID. The color code compares the individual row's distributions among clusters to the overall distribution among clusters reported in the last row. Clusters sorted by size. 
Furthermore, the partition of operation into recurrent delay patterns allows inference on individual clusters, which is not possible with association or succession rules [25-27]. These methods do not provide causality connection and can only be used to compare scenarios, for example, before and after delay mitigation countermeasures have been implemented. Results from clustering can be inferred with other mining techniques to identify further connections between specific system factors and delay membership, so that the causes of specific delays can be identified, and the effects of corrective actions can be estimated beforehand.

Alongside flexibility, the strength of this method resides in its simplicity. Unsupervised learning methods, such as clustering, aim at the identification of internal structures of the system. Supervised learning methods, in contrast, attempt to predict results, based on assumed connections in the input. For these reasons, neural networks [28, 30], Bayesian networks [29], and supporting vector regression methods [31] require initial assumptions on the factors that have direct effect on the desired output, which can be cumbersome to identify and could be hidden. The clustering method proposed here does not require initial assumptions, so any recurrent delay pattern can be identified. In particular, the $k$-means algorithm was selected, being the most common algorithm for partitional clustering. Even though several clustering methods and algorithms exist in the literature, none of them is clearly preferred to the others [33]. It is important to stress the fact that the output of clustering algorithms only suggests hypotheses and that the interpretation of results plays a more relevant role than seeking the best clustering principle. However, further research might improve the method. For example, a different choice of the clustering statistic between observations might be explored. In addition, the choice of the parameter $k$ might be supported by advanced techniques and metrics. In this paper, $k$ was set through statistical analysis of the associated clusters, but further studies might reveal more efficient methods integrated in the clustering algorithm itself. Lastly, the clustering results might depend on the punctuality threshold selected to filter out punctual trains, if applied.

The relations found in inference from resulting clusters can, eventually, be considered and implemented in the mentioned supervised data mining methods. The use of other sources of information can be further investigated, for example, the rolling stock equipment deployed or information on delay causes collected by train dispatchers. The clustering algorithm itself cannot provide information on the causes of delays, but relevant relationships with external variables might be found through the inference on the clusters. The implementation of information recorded by the dispatchers on primary and secondary delays could support the identification of delay propagation. However, previous studies in Europe highlighted the unreliability of such manually recorded data [11, 12]. These procedures are different for each infrastructure manager and should comply with different national regulations. This input should be analyzed in detail before being implemented in the inference on clusters. The timestamps might be integrated with data from other railway undertakings, so that the realized headways could be investigated and included in the cluster inferences. The effects of delay propagation might be thus investigated and the dispatching strategies possibly improved. Passenger counts or boarding/alighting timings could also reveal that specific localized delay increases are linked to passenger exchange and might suggest modifications in the scheduled dwelling times. Useful information from the railway undertakings might include differences between planned and realized train compositions or the use of energy saving strategies. Driving support systems are spreading among train operators to reduce the energy consumption and thus the operating cost, especially for diesel-powered railways. The effects of such systematic patterns in the driving style are, in any event, expected to emerge in the clustering algorithm, especially with more detailed data in the positioning. Further development of this method might expand its application to other industrial processes or other transportation modes. The service timekeeping could be measured at designated checkpoints, to build standard delay profiles and delay change profiles.

\section{Conclusions}

In this paper, a new method is presented to analyze railway operations, based on big data techniques. Previous studies highlighted the need for tools to analyze railway operation based on data from automatic data collection sources and to automatically detect delay patterns [1]. K-means clustering is here applied to train delay records from automatic train detection systems to identify systematic delays, rearranged in delay profiles and delay change profiles. This method is automatic, unbiased, flexible, and simple.

Both institutions and industry are showing great interest in big data applications [2]. The method described in this paper provides a managerial tool to identify recurrent delay patterns that affect the service reliability. A localized analysis with additional information supports the identification of the causes of individual patterns, so that specific countermeasures can be designed. For example, dispatching strategies might be modified when a structural conflict is detected; the boarding and alighting process might be improved at stations where delay increases recurrently. If the causes of recurrent delays are identified in frequent conflicts, small modifications to the timetable slack might be a solution to reduce delay propagation.

The effectiveness of this approach is demonstrated in an application on a Danish regional railway line. The application shows that it is possible to identify systematic delays at specific stations in a congested area and to identify different delay patterns. Furthermore, delay patterns can be conveniently associated with specific time periods of the day, showing time dependency, reasonably explained by the prevailing passenger flow direction. Specific delay patterns are demonstrated to be characteristic of individual train service IDs, which could depend on other service characteristics, such as structural conflicts with other trains in specific sections of the line, use of specific rolling stock equipment, or connections to other transport services. The implementation of other sources of information might improve the inference on the clusters, such 
as weather conditions, passenger counts, information from the dispatchers, or rolling stock characteristics.

Further development of this method might improve the selection of the number of clusters, identify new clustering metrics between observations, or integrate additional sources of information to improve the inference on clusters.

\section{Conflicts of Interest}

The authors declare that there are no conflicts of interest regarding the publication of this paper.

\section{Acknowledgments}

This work was funded by a Dean Grant from the Technical University of Denmark (DTU) and by the Danish Innovation Fund through the IPTOP (Integrated Public Transport Optimisation and Planning) project.

\section{References}

[1] B. Schittenhelm and T. Richter, "Railway Timetabling Based on Systematic Follow-up on Realized Railway Operations," in Proceedings of the Proceedings from the Annual Transport Conference at Aalborg University, H. Lahrmann, Ed., Aalborg: Traffic Research Group, Aalborg University, 2009.

[2] A. D’agostino, Big Data in Railways - Common Occurrence Reporting Programme, 2016, Big Data in Railways - Common Occurrence Reporting Programme.

[3] T. Richter, "En bedre jernbane gennem højere datakvalitet," in Trafikdage på Aalborgs Universitet. Aalborg: Traffic Research Group, H. Lahrmann, Ed., Aalborg University, 2008.

[4] A. Peterson, "Towards a robust traffic timetable for the swedish southern mainline," in Proceedings of the Computers in Railways XIII, pp. 473-484, WIT Transactions on The Built Environment, New Forest, UK.

[5] A. De Mauro, M. Greco, and M. Grimaldi, "A formal definition of Big Data based on its essential features," Library Review, vol. 65, no. 3, pp. 122-135, 2016.

[6] J. Hansen, "Future Railway Development and Performance," in Proceedings of the Danish Rail Conference, The Danish Rail Sector Association, Copenhagen, 2015.

[7] M. Carey, "Ex ante heuristic measures of schedule reliability," Transportation Research Part B: Methodological, vol. 33, no. 7, pp. 473-494, 1999.

[8] R. M. P. Goverde, G. Hooghiemstra, and H. P. Lopuhaä, Statistical Analysis of Train Traffic: The Eindhoven Case, IOS Press, Incorporated, 2001.

[9] H. Sipilä, "Simulation of modified timetables for high speed trains Stockholm - Göteborg," in Proceedings of the First International Conference on Road and Rail Infrastructure CETRA2010, S. Lakušić, Ed., p. 1, University of Zagreb, Opatija, Croatia, 2010.

[10] O. Lindfeldt, "Evaluation of punctuality on a heavily utilised railway line with mixed traffic," in Proceedings of the 11th International Conference on Computer System Design and Operation in the Railway and Other Transit Systems, COMPRAIL 2008, pp. 545-553, esp, September 2008.

[11] R. M. P. Goverde and L. Meng, "Advanced monitoring and management information of railway operations," Journal of Rail
Transport Planning and Management, vol. 1, no. 2, pp. 69-79, 2011.

[12] A. Ø. Sørensen, A. D. Landmark, N. O. E. Olsson, and A. A. Seim, "Method of analysis for delay propagation in a single-track network," Journal of Rail Transport Planning and Management, vol. 7, no. 1-2, pp. 77-97, 2017.

[13] A. Lindfeldt and H. Sipilä, "Simulation of freight train operations with departures ahead of schedule," in Proceedings of the 14th International Conference on Railway Engineering Design and Optimization, COMPRAIL 2014, pp. 239-249, Italy, June 2014.

[14] N. O. E. Olsson and H. Haugland, "Influencing factors on train punctuality - Results from some Norwegian studies," Transport Policy, vol. 11, no. 4, pp. 387-397, 2004.

[15] M. F. Gorman, "Statistical estimation of railroad congestion delay," Transportation Research Part E: Logistics and Transportation Review, vol. 45, no. 3, pp. 446-456, 2009.

[16] M.-C. Shih, C. T. Dick, S. L. Sogin, and C. R. L. Barkan, "Comparison of capacity expansion strategies for single-track railway lines with sparse sidings," Transportation Research Record, vol. 2448, pp. 53-61, 2014.

[17] A. Lindfeldt, "A study of the performance and utilization of the Swedish railway network," in Proceedings of the First International Conference on Road and Rail Infrastructure CETRA2010, S. Lakušić, Ed., University of Zagreb, Opatija, Croatia, 2010.

[18] T. Richter, "Systematic analyses of train run deviations from the timetable," in Proceedings of the 12th International Conference on Computer System Design and Operation in the Railways and other Transit Systems, COMPRAIL 2010, pp. 651-662, China, September 2010.

[19] E. Andersson, A. Peterson, and J. Törnquist Krasemann, "Robustness in Swedish Railway Traffic Timetables," in Proceedings of the 4th International Seminar on Railway Operations Modelling and Analysis, S. Ricci, I. A. Hansen, G. L. Longo, D. Pacciarelli, J. Rodriguez, and E. Wendler, Eds., pp. 1-18, Rome, 2011.

[20] B. H. Schittenhelm, "lanning With Timetable Supplements in Railway Timetables," in Proceedings of the Annual Transport Conference at Aalborg University, Aalborg, DK: trafikdage, 2011.

[21] E. Andersson, A. Peterson, and J. Törnquist Krasemann, "Introducing a New Quantitative Measure of Railway Timetable Robustness Based on Critical Points," in Proceedings of the 5th International Seminar on Railway Operations Modelling and Analysis (IAROR): RailCopenhagen2013, vol. 3, pp. 1-19, Copenhagen, 2013.

[22] N. van Oort, D. Sparing, T. Brands, and R. M. P. Goverde, "Data driven improvements in public transport: the Dutch example," Public Transport, vol. 7, no. 3, pp. 369-389, 2015.

[23] I. A. Hansen, R. M. P. Goverde, and D. J. Van Der Meer, "Online train delay recognition and running time prediction," in Proceedings of the 13th International IEEE Conference on Intelligent Transportation Systems, ITSC 2010, pp. 1783-1788, Portugal, September 2010.

[24] P. Kecman and R. M. P. Goverde, "Process mining of train describer event data and automatic conflict identification," in Computers in Railways XIII, WIT Transactions on the Built Environment, C. A. Brebbia, N. Tomii, P. Tzieropoulos, and J. M. Mera, Eds., vol. 127, pp. 227-238, 2012.

[25] B. Cule, B. Goethals, S. Tassenoy, and S. Verboven, "Mining train delays," Lecture Notes in Computer Science (including 
subseries Lecture Notes in Artificial Intelligence and Lecture Notes in Bioinformatics): Preface, vol. 7014, pp. 113-124, 2011.

[26] J. Wallander and M. Mäkitalo, "Data mining in rail transport delay chain analysis," International Journal of Shipping and Transport Logistics, vol. 4, no. 3, pp. 269-285, 2012.

[27] H. Yabuki, T. Ageishi, and N. Tomii, "Mining the Cause of Delays in Urban Railways based on Association Rules," in CASPT2015, pp. 1-16, 2015.

[28] G. Malavasi and S. Ricci, "Simulation of stochastic elements in railway systems using self-learning processes," European Journal of Operational Research, vol. 131, no. 2, pp. 262-272, 2001.

[29] P. Kecman, F. Corman, A. Peterson, and M. Joborn, "Stochastic prediction of train delays in real-time using Bayesian networks," in Proceedings of the in. Proceedings of the 13th Conference on Advanced Systems in Public Transport (CASPT, p. 18, Rotterdam (Netherlands), 2015.

[30] X. Chapuis, "Arrival Time Prediction Using Neural Networks," in 7th International Conference on Railway Operations Modelling and Analysis, N. Tomii, I. A. Hansen, J. Rodriguez, P. Pellegrini, S. Dauzère-Pérès, and D. De Almeida, Eds., pp. 1500-1510, International Association of Railway Operations Research, Lille, Fra, 2017.

[31] N. Marković, S. Milinković, K. S. Tikhonov, and P. Schonfeld, "Analyzing passenger train arrival delays with support vector regression," Transportation Research Part C: Emerging Technologies, vol. 56, pp. 251-262, 2015.

[32] P. Kecman and R. M. P. Goverde, "Predictive modelling of running and dwell times in railway traffic," Public Transport, vol. 7, no. 3, pp. 295-319, 2015.

[33] A. K. Jain, "Data clustering: 50 years beyond K-means," Pattern Recognition Letters, vol. 31, no. 8, pp. 651-666, 2010.

[34] H. Kashima, J. Hu, B. Ray, and M. Singh, "K-means clustering of proportional data using L1 distance," in Proceedings of the 2008 19th International Conference on Pattern Recognition (ICPR), pp. 1-4, Tampa, FL, USA, December 2008.

[35] T. Richter, A. Landex, and J. L. E. Andersen, "Precise and accurate train run data: Approximation of actual arrival and departure times," in Proceedings of the Precise and accurate train run data: Approximation of actual arrival and departure times, International Association of Railways, Sydney, Australia, 2013.

[36] T. Richter, Data aggregation for detailed analysis of train delays, C. A. Brebbia, N. Tomii, J. M. Mera, B. Ning, and P. Tzieropoulos, Eds., WIT Transactions on the Built Environment. WITPress, 2012.

[37] J. Wu, Advances in K-means Clustering, Springer, Berlin, Heidelberg, 2012.

[38] T. Hastie, R. Tibshirani, and J. Friedman, The Elements of Statistical Learning, Springer, New York, NY, USA, 2008. 


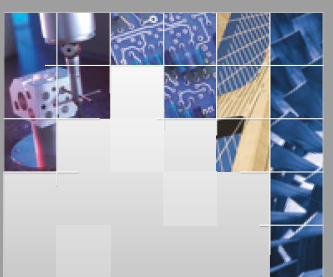

\section{Enfincering}
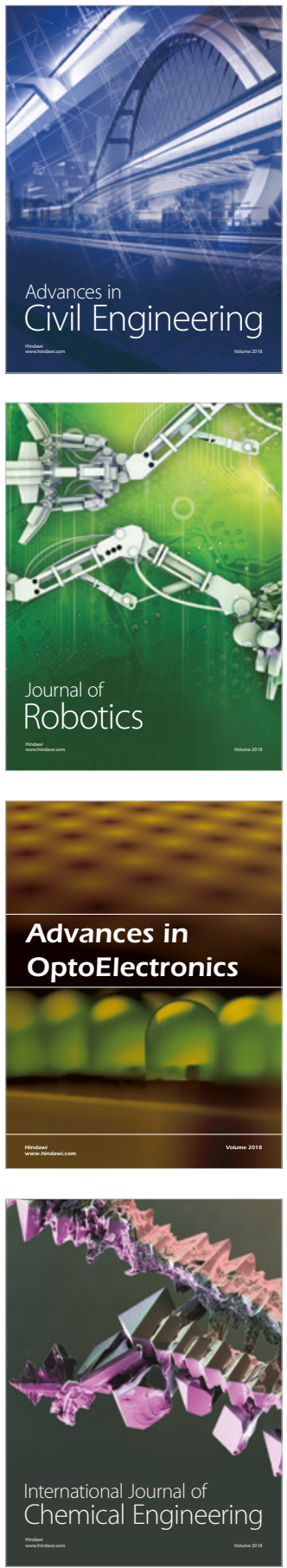

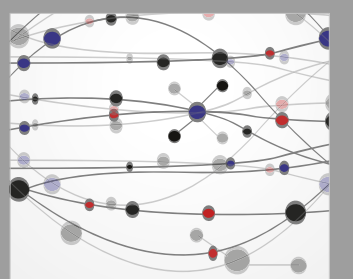

\section{Rotating \\ Machinery}

The Scientific World Journal

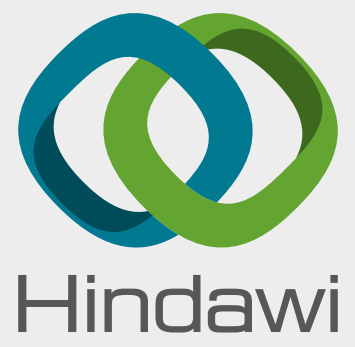

Submit your manuscripts at

www.hindawi.com
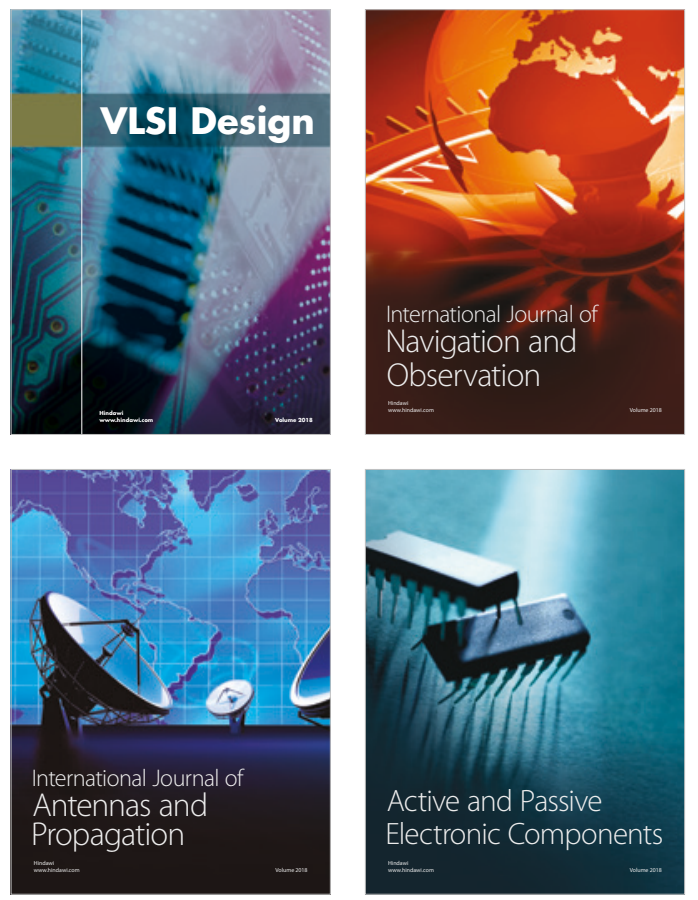
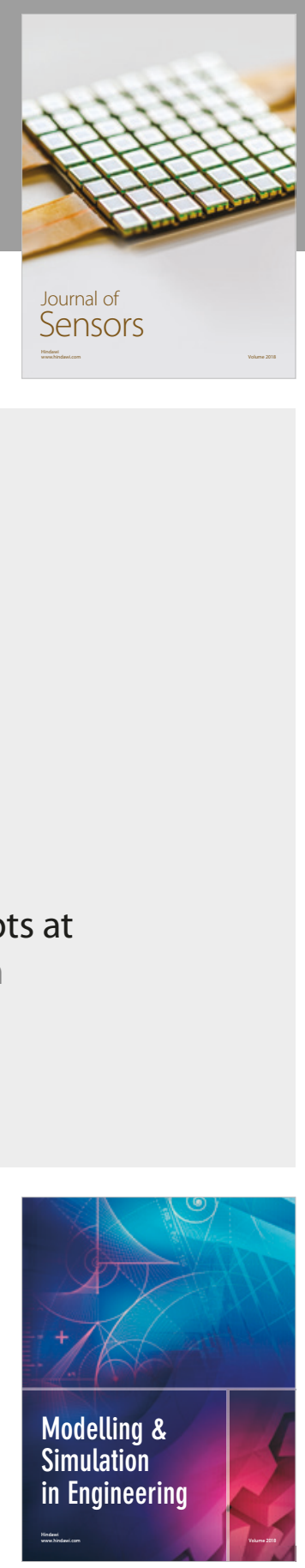

\section{Advances \\ Multimedia}
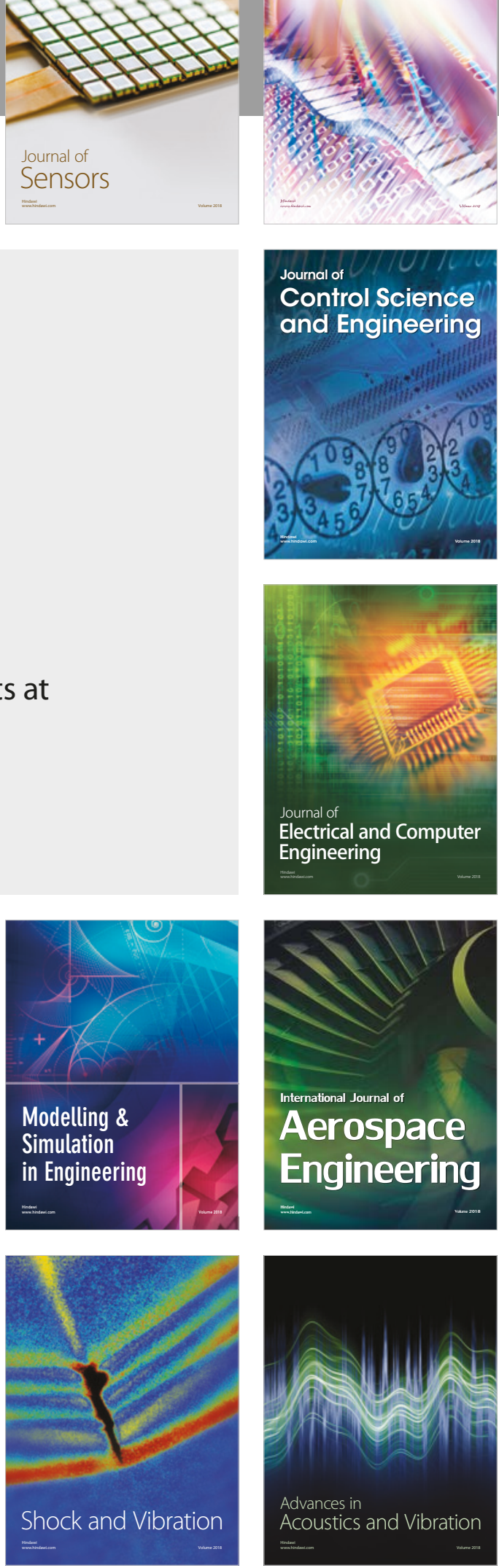